\title{
Spatial and transcriptional heterogeneity of $\beta$-cell neogenesis revealed by a time-resolved reporter system
}

\section{Shugo Sasaki}

BC Children's Hospital Research Institute

\section{Michelle Lee}

BC Children's Hospital Research Institute

\section{Yuka Wakabayashi}

Juntendo University Graduate School of Medicine

Luka Suzuki

Juntendo University Graduate School of Medicine

\section{Helena Winata}

BC Children's Hospital Research Institute

\section{Miwa Himuro}

Juntendo University Graduate School of Medicine

\section{Takaaki Matsuoka}

Osaka University

lichiro Shimomura

Osaka University Graduate School of Medicine

Hirotaka Watada

Juntendo University https://orcid.org/0000-0001-5961-1816

Francis Lynn

BC Children's Hospital Research Institute

Takeshi Miyatsuka ( $\nabla$ miyatsuka-takeshi@umin.net)

Juntendo University Graduate School of Medicine

\section{Article}

Keywords:

Posted Date: August 7th, 2020

DOI: https://doi.org/10.21203/rs.3.rs-46466/v1 
License: (c) (i) This work is licensed under a Creative Commons Attribution 4.0 International License. Read Full License 
Spatial and transcriptional heterogeneity of $\beta$-cell neogenesis revealed by a time-resolved reporter system

Shugo Sasaki ${ }^{1,2,3}$, Michelle Y. Y. Lee ${ }^{2}$, Yuka Wakabayashi ${ }^{4}$, Luka Suzuki ${ }^{4}$, Helena Winata ${ }^{2}$, Miwa Himuro $^{4}$, Taka-aki Matsuoka ${ }^{1}$, Iichiro Shimomura ${ }^{1}$, Hirotaka Watada ${ }^{4,5}$,

Francis C. Lynn ${ }^{1,2,3}$, Takeshi Miyatsuka ${ }^{4,5}$

${ }^{1}$ Department of Metabolic Medicine, Osaka University Graduate School of Medicine, Osaka, Japan

${ }^{2}$ Diabetes Research Group, BC Children's Hospital Research Institute, Vancouver, BC, Canada

3 Department of Surgery, School of Biomedical Engineering, University of British Columbia, Vancouver, BC, Canada

${ }^{4}$ Department of Metabolism and Endocrinology, Juntendo University Graduate School of Medicine, Tokyo, Japan

${ }^{5}$ Center for Identification of Diabetic Therapeutic Targets, Juntendo University Graduate School of Medicine, Tokyo, Japan

Corresponding authors:

Takeshi Miyatsuka and Francis C. Lynn

Department of Metabolism \& Endocrinology, Juntendo University Graduate School of Medicine, 2-

1-1 Hongo, Bunkyo-ku, Tokyo 113-8421, Japan

Tel: +81-3-5802-1579 / Fax: +81-3- 3813-5996

E-mail: miyatsuka-takeshi@umin.net (T.M.), francis.lynn@ubc.ca (F.C.L)

Main text (not including Abstract, Methods, References and figure legends): 3,830/5,000 words Title: 13/15 words, Abstract: 150/150 words, Methods: 1,524/3000 words, Figures/tables: 8/10 


\section{$1 \quad$ Abstract}

While pancreatic $\beta$ cells have been shown to originate from endocrine progenitors in ductal

3 regions, it remains unclear precisely where $\beta$ cells emerge and which transcripts define newborn $\beta$

4 cells. Here, we used a mouse model "Ins1-GFP;Timer" that provides spatial information during $\beta$ -

5 cell neogenesis with high temporal resolution. Fluorescent imaging demonstrated that, as expected,

6 some newborn $\beta$ cells arise close to the ducts; unexpectedly, all the others arise away from the ducts

7 and adjacent to blood vessels. Single-cell RNA-sequencing (scRNA-seq) demonstrated five distinct

8 populations of newborn $\beta$ cells, confirming the spatial heterogeneity of $\beta$-cell neogenesis, and

9 integration analysis with scRNA-seq of hESC-derived $\beta$-like cells uncovered transcriptional

10 similarity with the data in mouse $\beta$ cells. Thus, the combination of time-resolved histological imaging

11 with single-cell transcriptional mapping demonstrated novel features of spatial and transcriptional

12 heterogeneity in $\beta$-cell neogenesis, which will lead to a better understanding of $\beta$-cell differentiation

13 for future cell therapy.

\section{KEYWORDS}

$16 \quad \beta$ cell; differentiation; neogenesis; pancreatic duct; blood vessel; Mafa; Mafb; Gcg; single-cell

17 RNA-sequencing 


\section{Introduction}

Diabetes results from an absolute or relative deficiency of insulin-producing pancreatic $\beta$ cells, hence there is significant interest in developing safe and efficient protocols for the derivation of replacement $\beta$ cells. Understanding $\beta$-cell development provides clues towards a regeneration therapy for diabetes. To date, in vivo mouse models have uncovered hierarchical regulation of endocrine differentiation, showing that $\beta$ cells are differentiated from Neurog3-expressing endocrine progenitors that emerge from a progenitor compartment during embryogenesis ${ }^{1,2}$, and that endocrine neogenesis infrequently occurs in the adult pancreas under physiological conditions ${ }^{1-6}$. In addition, live imaging using Insulin 1 (Ins1)-GFP transgenic mice, which express enhanced green fluorescent protein (GFP) under the control of the Ins 1 promoter $^{7}$, demonstrated that individual $\beta$ cells migrate significant distances, forming islet-like clusters over time ${ }^{8}$. However, there is little spatial information describing precisely where $\beta$ cells arise from their endocrine progenitors, as there is no specific marker to distinguish newly generated $\beta$ cells separately from more differentiated $\beta$ cells.

Single-cell transcriptome analysis is a powerful new method to uncover cell characteristics in single cell resolution. In the field of pancreas development and diabetes, cell type characteristics and heterogeneity have been reported in healthy human pancreas ${ }^{9}$, the pancreas with type 2 diabetes ${ }^{10}$, human fetal pancreas ${ }^{11}$, pancreatic endocrine progenitors derived from human embryonic stem cells (hESCs) ${ }^{12}$, and mouse pancreatic endocrine cells ${ }^{13-15}$. However, single cell transcriptional profiling of $\beta$-cell neogenesis, downstream of endocrine differentiation, has not been reported.

We previously generated the "Ins1-Timer" reporter mouse model in which newly generated $\beta$ cells can be fluorescently separated from more differentiated $\beta$ cells using fluorescence activated cell sorting (FACS). Using this model, we subsequently determined the expression profiles of these two $\beta$-cell populations ${ }^{4}$. However, the fluorescence intensity of newborn $\beta$ cells in the Ins 1-Timer mice was below microscopic detection threshold (Figure 1A and Figure S1), and therefore the spatiotemporal characteristics of $\beta$-cell neogenesis could not be ascertained. To circumvent this 
43 problem, we developed a new mouse model, "Ins1-GFP; Timer", which provides the spatial

44 information of the newly generated $\beta$ cells with high intensity of green fluorescence, which can be

45 distinguished from more differentiated green/red double-fluorescent $\beta$ cells. Spatiotemporal imaging 46 with Ins 1-GFP; Timer mice demonstrated that $\beta$ cells are generated not only in close proximity to the 47 ductal lumen but also in an unexpected region that is away from the ducts and in close apposition to 48 blood vessels and pre-existing islets. Moreover, single-cell transcriptome analyses demonstrated five 49 distinct populations of newborn $\beta$ cells, and further confirmed the spatial heterogeneity of $\beta$-cell 50 neogenesis with unique distributions of MAFB- and GCG-expressing cells. Notably, single-cell RNA 51 profiles of hES-derived insulin-producing cells confirmed transcriptional heterogeneity during $\beta$-cell 52 neogenesis, which overlap with the cluster expression profiles of newborn mouse $\beta$ cells. Thus, this 53 new reporter system provides improved spatial and transcriptional information on newly generated $\beta$ 54 cells which, in turn, sheds light on novel microenvironments that may orchestrate endocrine 55 differentiation and $\beta$-cell neogenesis. 


\section{Results}

A novel reporter system for deciphering spatial heterogeneity during $\beta$-cell neogenesis

We previously developed reporter mouse models that express DsRed-E5 called "Fluorescent Timer", which shifts its fluorescence from green to red in a time-dependent manner ${ }^{16}$, allowing temporal separation of pancreatic cell lineages ${ }^{4,17}$. Among these reporter models, the "Insulin-Timer" mouse (Ins1-Timer), which expresses DsRed-E5 under the control of the Ins1 promoter, enabled us to separate newly generated $\beta$ cells from more differentiated $\beta$ cells by FACS. However, only green/red double-fluorescent cells were observed by microscopy, as the green fluorescence intensity of newly synthesized DsRed-E5 is below microscopic detection threshold in Ins 1-Timer embryos (Figure S1A). To overcome this limitation, another fluorescent protein, GFP, was simply introduced together with the DsRed-E5 reporter protein, by crossing Ins1-Timer mice with Insulin 1-GFP (Ins 1GFP) mice, in which the insulin 1 promoter drives GFP ${ }^{7}$. Because of the higher fluorescence intensity and faster maturation of Aequorea victoria GFP compared with DsRed-E5 ${ }^{16,18}$, we hypothesized that newly-specified $\beta$ cells would be bright green-dominant cells, which could be observed by fluorescence microscopy, in the Ins1-GFP; Timer double transgenic mice (Figure 1A and Figure S1A). As we had expected, green-fluorescent cells without red fluorescence were clearly observed by microscopy in the pancreas of Ins1-GFP; Timer double-transgenic mice, whereas only green/red double-fluorescent cells were observed in the pancreas of Ins1-Timer single-transgenic mice (Figure 1B). fluorescence as they mature, the pancreata of Ins1-GFP; Timer embryos were dissected and cultured ex vivo for real-time imaging of fluorescent cells. Time-lapse imaging with the pancreatic explants revealed that the green-fluorescent cells exhibited red fluorescence over time (Figure 1C). The period of time required for the transition from green-fluorescent cells to green/red double-fluorescent cells 
81 E5. As all fluorescent cells were stained with anti-insulin antibody (Figure S1B), these findings confirm that Ins1-GFP; Timer reporter mice are a useful tool for detecting and tracing newly generated $\beta$ cells using microscopy.

\section{Characterization of newborn $\beta$ cells by microscopy}

Microscopic quantification of fluorescent $\beta$ cells in Ins 1-GFP; Timer embryos demonstrated that $8.5 \%$ of whole fluorescent cells exhibited green fluorescence without red fluorescence at embryonic day 14.5 (E14.5), and the proportion of green-fluorescent cells gradually decreased at later stages of development (Figure 1D). There were few green-fluorescent cells at postnatal day 14, which was consistent with previous reports demonstrating that $\beta$-cell neogenesis rarely occurs after birth in mice 3,4,6,19. In contrast with Ins 1-Timer single-transgenic embryos, in which greater than $40 \%$ of $\beta$ cells were labeled as newly generated $\beta$ cells at E16.5 ${ }^{4}$, only $7.2 \%$ of $\beta$ cells exhibited green fluorescence in Ins1-GFP; Timer double-transgenic embryos at the same stage (Figure 1D), suggesting that an earlier $\beta$ cell population is labeled by Ins 1-GFP; Timer mice than Ins 1- Timer mice.

To investigate the characteristics of green-fluorescent cells, immunostaining for $\beta$-cell specific transcription factors was performed. All fluorescent cells were positive for pancreatic and duodenal homeobox 1 (PDX1) and NK6 homeobox1 (NKX6.1), which are known as essential transcription factors for $\beta$ cell development (Figures $2 \mathrm{~A}$ and $2 \mathrm{~B})^{20,21}$. In contrast, not all fluorescent cells were positive for musculoaponeurotic fibrosarcoma oncogene family A(MAFA), a transcription factor that was reported to be expressed exclusively in mature $\beta$ cells (Figures $2 \mathrm{C}$ and $2 \mathrm{D}$ ) ${ }^{22-24}$. The percentage 101 of MAFA-positive green-fluorescent cells was significantly lower than that of MAFA-positive green/red double-fluorescent cells between E16.5 and postnatal day 0 (Figure 2D). As MAFA was 103 shown to function downstream of NKX6.1 ${ }^{25}$, these results suggest that green-fluorescent cells are, not only temporally early but also, functionally immature $\beta$ cells. 
To investigate where $\beta$ cells are generated from their progenitors during development, the pancreata of Ins 1-GFP; Timer mice were stained with DBA lectin, a pancreatic duct marker (Figures $3 \mathrm{~A}-3 \mathrm{C}$ ), so that the relative position between green-fluorescent cells and ductal cells could be

110 determined. We confirmed previous reports demonstrating that endocrine cells arise in close 111 apposition with the ductal lumen ${ }^{1,17}$ as green-fluorescent newborn $\beta$ cells were observed adjacent to 112 the pancreatic ducts (Figure 3A, arrows). Unexpectedly, a significant number of green-fluorescent 113 cells were observed away from the ducts and were attached to green/red pre-existing $\beta$ cells (Figure $1143 \mathrm{~A}$, arrowheads). To exclude the possibility that green-fluorescent cells, which were observed away 115 from ductal cells on a thin section $(5 \mu \mathrm{m})$, are actually observed close to ductal cells on neighboring 116 sections, 3D images of 30-50 $\mu \mathrm{m}$-thick pancreata were acquired, and confirmed that there was some 117 distance between green-fluorescent $\beta$ cells and the ductal structures (Figure 3B).

To further determine the positional relationship between green-fluorescent cells and other cell types, immunostaining against PECAM1 was performed to label the endothelial cells within vessels, which demonstrated that many green-fluorescent cells located away from the ducts were in close contact with blood vessels (Figures $3 \mathrm{C}$ and S2). It was noted that none of the newborn $\beta$ cells were detected inside the islet-like clusters. Thus, spatiotemporal imaging with Ins1-GFP; Timer uncovered two different populations of newborn $\beta$ cells: 1) newborn " $\beta$ duct cells" near the ductal regions, and 2) newborn " $\beta^{\text {vessel }}$ cells" adjacent to both blood vessels and pre-existing islet-like clusters, suggesting two distinct locations of $\beta$-cell neogenesis. Quantitative analysis demonstrated that all the newborn $\beta$ cells consist of $\beta^{\text {duct }}$ and $\beta^{\text {vessel }}$ cells at all developmental stages after E14.5 (Figure 3D).

\section{Single-cell mRNA profiles uncovered temporal heterogeneity of newborn $\beta$ cells}

To further investigate transcriptional dynamics in early stage $\beta$ cells, single-cell RNAsequencing (scRNA-seq) was performed using E16.5 Ins 1-GFP; Timer mouse pancreas. Newborn $\beta$ 
131 cells and more differentiated $\beta$ cells were sorted as green-fluorescent cells and green/red double132 fluorescent cells, respectively (Figure 4A). Libraries of two different sets of green-fluorescent cells 133 (G1 and G2, 455 and 253 cells, respectively) and green/red double-fluorescent cells (GR, 1348 cells) 134 were sequenced at a depth of $191,716,144,968$ and 43,668 mean reads per cell, respectively. G1, G2 135 and GR cells were clustered, visualized and annotated using Seurat ${ }^{26,27}$ and t-distributed stochastic 136 neighbor embedding (t-SNE) ${ }^{28}$ as 12 individual clusters (Figure S3A). Out of 12 clusters, four 137 clusters were annotated as acinar, duct, endothelial and stellate clusters (375 cells, 18.2\%), based on 138 their expression profiles (Table S1) and eight other clusters were found to robustly express Ins 1 and 139 Ins2 (1,681 cells, 81.8\%) (Figure S3B and S3C). Notably, unbiased clustering resulted in clear 140 difference in distribution between green-fluorescent cells and green/red double-fluorescent cells 141 (Figure 4B). Pseudotime trajectory analysis, generated with Monocle ${ }^{29,30}$, demonstrated that green142 fluorescent cells (G1 and G2) were enriched at the beginning of pseudotime as an earlier cluster on 143 pseudo-temporal axis (Figure 4C, 4D, and S3D). In addition, single-cell expression levels of Ins 1 and 144 Ins 2 over pseudotime clearly showed sequential increase in Ins1 and Ins 2 mRNAs as $\beta$ cells 145 differentiate (Figure S3E and S3F). These findings confirmed high consistency between 146 computational pseudotime trajectory and the temporal shift of fluorescent spectrum designed by two 147 reporter transgenes (Figure 1A).

148 To decipher detailed transcriptional dynamics in $\beta$-cell neogenesis, we focussed on green149 fluorescent newborn $\beta$ cells (G1 and G2), which were sequenced more deeply than green/red double150 fluorescent cells (GR). After exclusion of non-endocrine lineage cells (Figure S4A and S4B), green151 fluorescent newborn $\beta$ cells were subclustered into five groups, which were named as $G^{\text {Neurog3 }}, G^{S s t}$, $152 G^{G c g}, G^{P d x l}$, and $G^{I n s 2}$, based on the most differentially expressed gene in each cluster (Fig 4E, 4F, and $153 \mathrm{~S} 5 \mathrm{~A}$ ). To reconstruct an unbiased lineage trajectory for these five subpopulations during $\beta$-cell 154 neogenesis, pseudotime analysis was performed and demonstrated that $G^{S s t}$ cells were located around 155 bifurcation from $G^{\text {Neurog3 }}$ cells into $G^{P d x 1}$ and $G^{I n s 2}$ cells or $G^{G c g}$ cells (Figure 5A, 5B). Furthermore, 
branched expression analysis modelling (BEAM) clarified transcriptional dynamics along pseudotemporal trajectories (Figure 5C and S5B-S5D). Among top 100 differentially expressed genes in BEAM, some transcriptions factors, which have been shown to play pivotal roles in endocrine differentiation, were extracted. For example, $M a f b$ and $I s l l$ were highly expressed at the intermediate regions of $G^{P d x 1} / G^{I n s 2}$ and $G^{G c g}$ branch, respectively. In contrast, Arx and $\operatorname{Irx} 2$, which are known to be

161 expressed in $\alpha$ cells, showed the peak expression at the end of $G^{G c g}$ branch (Figure 5C, and S5B). 162 Taken together with temporal dynamics of Ins 2 and Gcg mRNAs showing the relative low expression 163 high temporal resolution.

\section{Transcriptional heterogeneity during $\beta$-cell maturation}

Ins1-GFP; Timer mouse model allows us to label not only newborn $\beta$ cells but also more differentiated $\beta$ cells (Figure 4A). To investigate gene sets important for $\beta$-cell maturation, green/red double fluorescent cells were analysed by scRNA-seq. Unbiased annotation revealed six distinct clusters in more differentiated $\beta$ cells (Figure 6A, 6B). The trajectory given by Monocle showed one directional development toward $G R^{S c g 2}$ cells expressing Scg2, a member of the chromogranin family, and carboxypeptidase E (Cpe), which is an key enzyme for proinsulin processing (Figure 6C, 6D) ${ }^{31}$. Notably, the expression profiles of $G R^{S p c 25}$ cluster shared key characteristics of proliferating cells and 
181 lacked markers of cells in G1 stage following cell cycle analysis (Figure 6B, 6E). The pseudotime 182 analysis showed a unique branch consisting of $G R^{S p c 25}$ cells adjacent to $G R^{N p y}$ cluster, which is located 183 prior to most mature $G R^{S c g 2}$ cluster (Figure 6C). In contrast, unbiased annotation with green184 fluorescent newborn $\beta$ cells did not annotate proliferating insulin-positive population (Figure 4E, 4F, $1855 \mathrm{~A}, \mathrm{~S} 4 \mathrm{~A}$ and $\mathrm{S} 4 \mathrm{~B})$. These findings suggest that $\beta$-cell proliferation is tightly regulated during a 186 specific time window during $\beta$-cell differentiation, which is consistent with our previous finding with Ins 1-Timer embryos ${ }^{4}$.

\section{Orchestration between spatial and transcriptional heterogeneity during $\beta$-cell neogenesis}

Histological imaging in Ins 1-GFP; Timer embryos resulted in two distinct populations (i.e. $\beta^{\text {duct }}$

and $\beta^{\text {vessel }}$ cells) during $\beta$-cell neogenesis, whereas unbiased clustering with single-cell mRNA profiles demonstrated five clusters $\left(G^{\text {Neurog3 }}, G^{S s t}, G^{G c g}, G^{P d x l}\right.$, and $\left.G^{\text {Ins2 }}\right)$. To investigate whether transcriptional heterogeneity was indeed observed between $\beta^{\text {duct }}$ and $\beta^{\text {vessel }}$ cells, immunostaining was performed with antibodies against NEUROG3, SST, GCG, and MAFB, mRNAs of which were highly expressed in $G^{\text {Neurog3 }}, G^{S s t}, G^{G c g}$, and $G^{P d x l}$, respectively (Figure 4F). NEUROG3 and SST proteins did not overlap with either green or red fluorescent cells (data not shown). Immunostaining against GCG demonstrated that $56 \%$ and $28 \%$ of newborn $\beta^{\text {duct }}$ cells in Ins 1-GFP; Timer embryos were positive for GCG at E14.5 and E16.5, respectively (Figure 7A and 7B), whereas none of $\beta^{\text {vessel }}$ cells were positive for GCG at either stage, suggesting that $G^{G c g}$ cells comprise part of $\beta^{\text {duct }}$ cells. Furthermore, immunostaining against MAFB demonstrated that more than $90 \%$ of $\beta^{\text {duct }}$ cells were positive for MAFB, while only $20 \%$ of $\beta^{\text {vessel }}$ cells were labeled (Figure 7C and 7D). These findings suggest that most of $G^{P d x l}$ cells, which highly express $M a f b$, belong to $\beta^{\text {duct }}$ cells, while only part of $G^{P d x I}$ cells compose $\beta^{\text {vessel }}$ cells. Thus, since the expression pattern of MAFB uncovered unique spatial heterogeneity of newborn $\beta$ cells, we next examined the immunostaining for MAFA, another member of MAF family transcription factor, which was detected in part of green-fluorescent cells (Figure 2C 
and 2D), although Mafa transcripts were not sequenced in our scRNA-seq data. Intriguingly, the expression patterns of MAFA were opposite to those of MAFB (Figure 7E, 7F); more than $80 \%$ of $\beta^{\text {vessel }}$ cells were positive for MAFA, significantly higher than that of $\beta^{\text {duct }}$ cells, in which less than $30 \%$ of them expressed MAFA. The expression patterns of these three key proteins imply that $\beta^{\text {vessel }}$ cells are functionally more mature than $\beta^{\text {duct }}$ cells despite having an identical time window after the Ins1 promoter is activated. Considering that MAFA is thought to function as $\beta$ cells mature, and the pseudotime trajectory showed that $G^{I n s 2}$ cells are more differentiated than $G^{P d x l}$ cells, these findings suggest that $G^{\text {Ins } 2}$ cells may comprise MAFA-expressing $\beta^{\text {vessel }}$ cells. Thus, the histological analysis of newborn $\beta$ cells accompanied by single-cells mRNA profiles demonstrated a unique orchestration between spatial and transcriptional heterogeneity during $\beta$-cell neogenesis.

\section{Human embryonic stem cell-derived $\beta$-like cells share mouse newborn $\beta$-cell features}

To date, in vitro differentiation protocols of human embryonic stem cells (hESCs) toward $\beta$-like cells have been developed, although the differentiated cells induced by most of the protocols are a mixed population of $\sim 50 \%$ insulin-producing cells and $\sim 50 \%$ of other cell types ${ }^{32-34}$. Additionally, these $\beta$-like cells are still immature because they need in vivo transplantation to give rise to mature $\beta$ cells that have the same glucose-responsive insulin secretion capacity as that of bona fide $\beta$ cells. To investigate similarity between hESC-derived immature $\beta$-like cells and mouse newborn $\beta$ cells, transcriptional profiles were compared using scRNA-seq data. Cyt49 hESCs were differentiated for 27 days in vitro by a protocol reported by Nair et al. ${ }^{32}$, and 5,644 cells were analyzed after sequencing and filtering. After running Seurat, the clusters which contained 1,107 INS- highly expressing cells ( $20 \%$ of total cells) were selected and re-clustered to 7 clusters visualized on t-SNE (Figure $8 \mathrm{~A}-8 \mathrm{C}$ ). $M G S T 1$ and SYT13 are enriched in $h \beta^{M G S T 1}$ and $h \beta^{S Y T 13} \beta$-like populations, respectively, consistent to that mouse Mgst 1 and Syt13 have the peak expression in very early $\beta$ cells (Figure S5D). Furthermore, 
$231 h \beta^{N E F M}, h \beta^{I A P P}, h \beta^{G A S T}$, and $h \beta^{G C G}$ clusters (Figure 8D, 8E), as $S s t$-expressing $G^{S s t}$ cluster was located 232 at an earlier stage than $G^{P d x l}, G^{I n s 2}$, and $G^{G c g}$ clusters (Figure 5A).

233 To further investigate transcriptional relevance between mouse $\beta$ cells and hESC-derived $\beta$-like 234 cells, the integration analysis was performed with data sets from mouse $\beta$ cells from Ins 1-GFP; Timer 235 embryos and hESC-derived $\beta$-like cells (Figure S6A-S6C). Although the integrated t-SNE clustering 236 showed few overlaps between mouse and human cells, Neurog3-, Gcg- and Sst-expressing 237 populations in mouse embryos appeared in distinct areas that were overlapped with human cells by 238 more than $15 \%$ distribution in each cluster (Figure S6D, S6E), suggesting that only $\beta^{\text {Neurog3 }}, \beta^{\text {Gcg }}$ and $239 \quad \beta^{S s t}$ share transcriptional profiles with NEUROG3-, GCG- and SST-expressing human cells. Thus, 240 comparative analysis of hESC-derived $\beta$-like cells with mouse newborn $\beta$ cells indicates potential 241 shared transcriptional dynamics during early $\beta$-cell specification that remain to be further elucidated. 


\section{Discussion}

Advances in imaging technology using fluorescent proteins both in vivo and ex vivo have enabled

244 us to capture cellular dynamics that occur during pancreas development and $\beta$-cell differentiation ${ }^{7,35}$.

245 While reporter mouse models that express fluorescent proteins have helped to trace the differentiation

246 of specific cell types in endocrine lineages, it remains to be established as to how the newly specified

$247 \beta$ cells can be detected by microscopy separately from more differentiated $\beta$ cells, due to the lack of

248 a specific marker for early $\beta$-cell neogenesis. In the present study, we demonstrated that a combination

249 of two fluorescent proteins under the same Ins 1 promoter provides a discrete time window to address

250 this difficulty, and we successfully demonstrated the two distinct pancreatic regions from which $\beta$ 251 cells arise.

Previously a Cre/loxP-mediated reporter system combined with microscopy was used to show no evidence of $\beta$-cell neogenesis in adult pancreata ${ }^{19}$, which agrees with our findings with Ins 1-Timer

$254{ }^{4}$ and Ins1-GFP; Timer mice (Figures 1D). On the other hand, at E14.5, Cre/loxP-mediated reporter mice labeled all the insulin-producing cells as a single population, whereas Ins1-GFP; Timer embryos clearly demonstrated temporal heterogeneity of $\beta$-cell differentiation; that is, around $8.5 \%$ of insulinproducing cells were labeled as green-fluorescent, newly differentiated $\beta$ cells, which were clearly 258 distinguished from green/red double-fluorescent, more differentiated $\beta$ cells (Figures 1D). This 259 difference is probably because the temporal resolution in the Cre/loxP reporter mice depended on the 260 degradation of red fluorescent protein, which has a longer half-life, whereas our mouse model takes 261 advantage of the shorter time window caused by the higher intensity and faster maturation of GFP 262 than DsRed-E5 (Figures 1A and S1A). Thus, the short time window in Ins1-GFP; Timer embryos 263 could provide novel insights into spatio-temporal heterogeneity during $\beta$-cell neogenesis. Fluorescence imaging with Ins 1-GFP; Timer embryos demonstrated the existence of two types 265 of newborn $\beta$ cells: " $\beta$ duct cells" near the pancreatic ducts and " $\beta$ vessel cells" near the blood vessels 266 (Figures 3A-3C). As Neurog3-expressing endocrine progenitors emerge from the ductal region and 
give rise to hormone-expressing endocrine cells ${ }^{1,2}$, we were not surprised to see Neurog3-expressing cells differentiate into $\beta^{\text {duct }}$ cells near the ductal compartment without further migration. In contrast, the existence of $\beta^{\text {vessel }}$ cells and the lack of green-fluorescent cells in every other region between pancreatic ducts and blood vessels supports the possibility that endocrine progenitors migrate away

271 from the ductal area toward the region near the blood vessels prior to differentiation (Figure S7). On 272 the other hand, it has been reported that Neurog3-expressing cells were observed in epithelial tip 273 domains as well as in trunk domains ${ }^{36}$, implying that $\beta^{\text {vessel }}$ cells might emerge from Neurog3expressing progenitors in the tip domains. While several mouse models have been developed to study the regulatory mechanisms of islet cell migration ${ }^{37,38}$, it remains unclear when and how $\beta$ cells are specified during migration. It would be of great interest to trace the emergence of $\beta^{\text {duct }}$ and $\beta^{\text {vessel }}$ cells under the circumstances where islet cell migration is disrupted in these mouse models.

Blood vessels and their surrounding environments have been demonstrated to play essential roles in various stages of pancreas development. For example, aortic endothelial cells are required for the maintenance of Pdx 1 expression and for pancreas budding ${ }^{39}$, and ectopic vascularization leads to ectopic insulin expression ${ }^{40}$. The presence of $\beta^{\text {vessel }}$ cells suggests that vascular endothelial cells may endow endocrine progenitors with a $\beta$-cell destiny. Further studies would be needed to address the microenvironment around $\beta^{\text {duct }}$ and $\beta^{\text {vessel }}$ cells, which may lead to the discovery of new substances critical for $\beta$-cell specification.

We performed single-cell transcriptome analysis with Ins 1-GFP; Timer embryos and found that unbiased clustering resulted in five unique populations among green-fluorescent newborn $\beta$ cells. 287 Since $M a f b$ mRNAs were differentially expressed in $G^{P d x l}$ cells (Figure 4F) and MAFB proteins were 288 detected in a larger number of $\beta^{\text {duct }}$ cells than in $\beta^{\text {vessel }}$ cells, it is possible that $M a f b$-expressing $G^{P d x I}$ 289 cells may compose MAFB-expressing $\beta^{\text {duct }}$ cells. However, there are some difficulties to validate the 290 correlations between five populations annotated by scRNA-seq and two populations observed on 291 microscopy. For example, neither SST nor NEUROG3 overlapped with fluorescent proteins probably 
292 because the detection threshold of immunostaining was not sufficiently high. On the other hand, some 293 transcripts like Mafa were not sequenced in scRNA-seq, although MAFA proteins were clearly 294 detected (Figure 2C and 7E). Technical improvement in scRNA-seq methodologies, including 295 improved sequencing depths, would advance our understanding concerning transcriptional 296 heterogeneity during $\beta$-cell neogenesis.

297 Single-cell transcriptome analysis in hESC-derived $\beta$-like cells identified $S S T$-expressing $h \beta^{S S T}$ 298 cells as an earlier cluster (Figure 8A-8E), showing similarity with mouse $S s t$-expressing $G^{S s t}$ cluster 299 in newborn $\beta$ cells which was located at an earlier stage than some other clusters. In addition, the 300 integration analysis between Ins 1-GFP; Timer embryos and hESC-derived $\beta$-like cells confirmed 301 shared transcriptional profiles among Sst/SST-expressing subsets (Figure S6D, S6E). These findings 302 suggest that Sst-expressing cells are in an intermediate state during $\beta$-cell maturation in both mouse 303 and human, which is consistent with recent findings that hESC-derived $\beta$-like cells co-expressed SST 304 transcripts during differentiation ${ }^{12}$.

305 In summary, this time-resolved reporter system in mice revealed spatial and transcriptional 306 heterogeneity during $\beta$-cell neogenesis, showing similarity and difference between mouse $\beta$ cells and 307 hESC-derived $\beta$-like cells. Although these novel findings lead to new additional questions that should 308 be addressed, further time-resolved comprehensive analyses at the single-cell level will provide us 309 with novel insights, which could be applied to regeneration therapy for diabetes. 
Animals

Ins1-Timer and Ins1-GFP reporter mice were generated as previously described ${ }^{4,7}$. Ins 1GFP; Timer double-transgenic mice were obtained by crossing Ins 1-Timer mice with Ins1-GFP mice. 314 The mice were genotyped by PCR, using the forward primer 5'-AGTTCCAGTACGGCTCCAAG-3' 315 and the reverse primer 5'-CAGCCCATGGTCTTCTTCTG-3' in the coding region of DsRed-E5 for Ins 1-Timer and the forward primer 5'-GAACTTCAAGATCCGCCACA-3' and the reverse primer 5'ACTCCAGCAGGACCATGTGA-3' in the coding region of GFP for Ins 1-GFP, respectively. Timed mating was carried out with E0.5 being set as midday of the day of discovery of a vaginal plug. Mice were housed on a 12-h light-dark cycle in a controlled climate. All animal procedures were approved by the Ethics Review Committee for Animal Experimentation of Osaka University Graduate School of Medicine, and Juntendo University Graduate School of Medicine.

\section{Histological analyses}

Tissues were fixed in $4 \%$ paraformaldehyde in phosphate buffered saline (PBS) at $4{ }^{\circ} \mathrm{C}$, washed in PBS, immersed in sucrose solution, and embedded in Tissue-Tek OCT Compound (Sakura, Japan). Frozen blocks were sectioned at $5-\mu \mathrm{m}$ thickness for regular observation and at $30-\mu \mathrm{m}$ thickness for 3D analysis. Sections were blocked with 5\% horse serum, incubated with primary antibodies overnight at $4{ }^{\circ} \mathrm{C}$, and then visualized by incubation with secondary antibodies for $1 \mathrm{hr}$ at room temperature. The primary antibodies used were the following: chicken anti-insulin $(1: 1,000$; Abcam, 330 Cambridge, MA, USA), rabbit anti-glucagon (1:1,000; Dako, Carpinteria, CA, USA), guinea pig anti331 PDX1 (1:500; a kind gift from Dr. M. German) rabbit anti-NKX6.1 (1:200; Sigma-Aldrich, St Louis, 332 MO, USA), rabbit anti-MAFA (1:500; Bethyl Laboratories, Montgomery, TX, USA), rat anti333 PECAM1 (1:250; BD Biosciences, San Jose, CA, USA) and biotinylated lectin Dolichos biflorus 334 agglutinin (DBA; 1:200; Vector Laboratories, Burlingame, CA, USA). The secondary antibodies used 
were Alexa Fluor 647-conjugated anti-rabbit IgG, Alexa Fluor 647-conjugated anti-guinea pig IgG, Alexa Fluor 647-conjugated anti-rat IgG, Alexa Fluor 647 and 350-conjugated streptavidin, Alexa Fluor 350-conjugated anti-rabbit IgG, and DyLight 350-conjugated anti-chicken IgY (all at 1:200; Invitrogen, Carlsbad, CA, USA). After washing in PBS, sections were mounted in Vectashield mounting medium (Vector Laboratories). Images were acquired using an Olympus FV1000D 340 confocal laser scanning microscope and a Keyence BZ-9000 all-in-one fluorescence microscope. For morphometric analysis, the number of fluorescent or immunostained cells was quantified (more than three sections for each mouse) with Image J software (NIH, Bethesda, MD, USA).

\section{Ex vivo real-time imaging of the developing pancreas}

Developing pancreata were dissected from Ins1-GFP; Timer embryos at E14.5, and were stored in sterile PBS on ice until all dissections were completed. For growth, organ explants were transferred to coverglass-bottomed dishes (AGC Asahi Glass, Tokyo, Japan) coated with $30 \mu \mathrm{L}$ Matrigel ${ }^{\mathrm{TM}}$ (BD Biosciences, San Jose, CA, USA), and then incubated with growth medium (DMEM/F-12 with 10\% FBS, antibiotics, and insulin-transferrin-selenium). Live imaging of the explants was carried out using an Olympus FV1000D confocal laser-scanning microscope, with an automated motorized stage, in a humidified heated chamber with $\mathrm{CO}_{2}$. For acquisition, digital images of 12 sections per z-stack were captured through multiple z-steps (5- $\mu \mathrm{m}$ apart) every $30 \mathrm{~min}$. The total time of imaging was 10 hr. After imaging, z-stacks were collapsed into maximum intensity projections, and all time points were compiled and exported as a QuickTime (avi) file using Image J software (NIH, Bethesda, MD, USA).

\section{Sorting cells for single-cell RNA-sequencing}

Ins1-GFP; Timer embryos were collected on E16.5 and dissected on ice. To generate single cells, embryonic pancreases were incubated in $2 \mathrm{~mL}$ of pre-warmed $37{ }^{\circ} \mathrm{C} 0.25 \%$ Trypsin with mild 
agitation for 5 minutes. To stop digestion, $1 \mathrm{~mL}$ of cold FBS and $2 \mathrm{~mL}$ of cold PBS were added and mixed by inversion to stop digestion, followed by pipette filtering with a $40 \mathrm{~mm}$ nylon filter. Cells were then centrifuged for 5 minutes at $200 \mathrm{~g}$. After aspirating the supernatant, cells were resuspended in cold $2 \%$ FBS in PBS, placed on ice, and immediately sorted into green-fluorescent, and green/red double-fluorescent fractions using a Beckman Coulter MoFlo Astrios (Mississauga, ON, Canada).

\section{Preparation of human $\beta$-like cells for single-cell RNA-sequencing}

CyT49 parental hESC line was obtained from ViaCyte, Inc. (San Diego, CA, USA). Studies with undifferentiated CyT49 hESCs were plated on six-well plates at a density of 5.5 million cells in 5.5 $\mathrm{mL}$ media per well. The plates were incubated at $37^{\circ} \mathrm{C}$ and $5 \% \mathrm{CO} 2$ on an orbital shaker at $100 \mathrm{rpm}$ to induce spheroid formation. After $24 \mathrm{~h}$, seven-step differentiation was induced. Stage 7 cells were differentiated longer than the original protocol, and differentiation was stopped at day $27 . \sim 100$ spheroids were washed once with PBS before adding $500 \mu \mathrm{L}$ of Accutase. Following $10 \mathrm{~min}$ at $37^{\circ} \mathrm{C}$ for dissociation, $500 \mu \mathrm{L}$ of $2 \%$ BSA CMRL medium was added, centrifuged for 5 min at $200 \times \mathrm{g}$, washed once with PBS, and resuspended in $350 \mu \mathrm{L}$ of ice-cold PBS. USA) as previously described ${ }^{15}$. The Single Cell 3' Reagent Kits v2 were used to generate single cell libraries. Briefly, cells were counted following FACS and cell suspensions were loaded for a targeted cell recovery of 1000-5000 cells per channel. The microfluidics platform was used to barcode single 
cDNA from single cells. The full length, barcoded cDNA is PCR amplified followed by enzymatic fragmentation and SPRI double sided size selection for optimal cDNA size. End repair, A-tailing, Adaptor Ligation, and PCR are performed to generate the final libraries that have P5 and P7 primers compatible with Illumina sequencing. The libraries were pooled and sequenced using an Illumina NextSeq500 platform with a 150 cycle High Output v2 kit in paired-end format with 26 bp Read 1, 8 bp I5 Index, and 85 bp Read 2.

\section{Data Analysis}

Following sequencing, data were analysed using publicly available software programs and $\mathrm{R}$ pipelines. First, cellranger mkfastq (10x Genomics) generates FASTQ files from the raw sequencing data. Next, cellranger count uses the FASTQ file to perform sequence alignment (mouse: GRCm38, human: GRCh38), filter sequences based on quality score, and generate single cell gene counts. For count alignment of the lineage-tracing transgenes, the mouse reference genome was annotated to include the GFP and Timer sequences. Cellranger aggr was used to combine data from multiple samples (G1, G2 and GR) and ensure all libraries have the same sequencing depth.

As minimal filtering is performed in cellranger, two additional $\mathrm{R}$ pipelines were used to filter 401 out cells that did not meet the quality control standard. The first pipeline is Scater 402 (https://bioconductor.org/packages/release/bioc/html/scater.html) and is a single cell analysis pipeline that places a great emphasis on quality control ${ }^{41}$. For this analysis, cells were discarded based on counts (transcripts/cell), genes (genes/cell) and percentage mitochondrial DNA expression, greater than 3 standard deviation away from the mean. Low-abundance genes, which were expressed by less 406 than 3 cells, were removed. This QC dataset was then analyzed using the Seurat V2.0 and V3.0 407 pipeline (http://satijalab.org/seurat/), another R toolkit for single cell genomics ${ }^{26,27}$. Seurat posed an additional filtering step that removed out cells with less than 500 genes. Seurat was then used for normalization, correcting cells by their library size, and linear dimensional reduction, removing 
uninteresting source of variations. Finally, unsupervised shared nearest neighbor modularity

411 optimization based clustering was performed using Seurat to group cells based on gene expression 412 and to identify unique cell types within the populations. For cell cycle analysis, cell-cycle scoring 413 and phase determination pipelines provided with Seurat was assigned to the GR library. Cross-species 414 integration analysis was conducted using Seurat V3.0.

\section{Pseudotime analysis}

Pseudotime analysis was performed using Monocle v2.6.1 (http://cole-trapnell-lab.github.io/ monocle-release/docs/\#constructing-single-cell-trajectories). Quality controlled transcript data by

419 Scater and Seurat were loaded into Monocle as a CellDataSet object. Same filtering threshold was 420 used as Seurat, in while cells with less than 500 genes, and genes expressed by less than 3 cells were 421 removed. Variable expressed genes were defined as a gene that was expressed in $>50$ cells. 422 Unsupervised clustering was performed using genes that have a mean expression of $>0.1$ and 423 dimensional reduction was done using t-SNE. Next, differential gene expression analysis was done 424 between clusters of interest and the top 1000 variable genes with qval $<0.01$ were used to learn a tree425 like trajectory using DDRTree method and lastly order cells in the pseudotime. Branched expression analysis modelling (BEAM) provided with Monocle was conducted to determine gene expression patterns over pseudotime.

\section{Statistical analyses}

Measurements were performed on discrete samples unless otherwise stated. Statistical analyses 431 were performed using the GraphPad Prism 8.0 and R software. Comparisons of two samples were 432 performed by the unpaired two-tailed $t$-tests. Multiple groups were analysed by one-way ANOVA 433 with a multiple comparison test and the Tukey-Kramer's post-hoc test was used to compare different 
434 groups. A value of $p<0.05$ was considered to indicate a statistically significant difference between 435 two groups. Data are presented as the mean \pm SEM. 


\section{References}

437 1. Schwitzgebel, V. M. et al. Expression of neurogenin3 reveals an islet cell precursor population in the pancreas. Development 127, 3533-42 (2000).

2. Gu, G., Dubauskaite, J. \& Melton, D. A. Direct evidence for the pancreatic lineage: NGN3+ cells are islet progenitors and are distinct from duct progenitors. Development 129, 2447-57 (2002).

3. Dor, Y., Brown, J., Martinez, O. I. \& Melton, D. A. Adult pancreatic beta-cells are formed by selfduplication rather than stem-cell differentiation. Nature 429, 41-6 (2004).

4. Miyatsuka, T. et al. Chronological analysis with fluorescent timer reveals unique features of newly generated $\beta$-cells. Diabetes 63, 3388-93 (2014).

5. Solar, M. et al. Pancreatic exocrine duct cells give rise to insulin-producing beta cells during embryogenesis but not after birth. Dev Cell 17, 849-60 (2009).

6. Teta, M., Rankin, M. M., Long, S. Y., Stein, G. M. \& Kushner, J. A. Growth and regeneration of adult beta cells does not involve specialized progenitors. Dev Cell 12, 817-26 (2007).

7. Hara, M. et al. Transgenic mice with green fluorescent protein-labeled pancreatic beta -cells. Am J Physiol Endocrinol Metab 284, E177-83 (2003).

8. Puri, S. \& Hebrok, M. Dynamics of embryonic pancreas development using real-time imaging. Dev Biol 306, 82-93 (2007).

9. Enge, M. et al. Single-Cell Analysis of Human Pancreas Reveals Transcriptional Signatures of 
455 10. Segerstolpe, A. et al. Single-Cell Transcriptome Profiling of Human Pancreatic Islets in Health $456 \quad$ and Type 2 Diabetes. Cell Metab 24, 593-607 (2016).

457 11. Ramond, C. et al. Understanding human fetal pancreas development using subpopulation sorting, $458 \quad$ RNA sequencing and single-cell profiling. Development 145, (2018).

459 12. Petersen, M. B. K. et al. Single-Cell Gene Expression Analysis of a Human ESC Model of 460 Pancreatic Endocrine Development Reveals Different Paths to beta-Cell Differentiation. Stem 461 Cell Rep. 9, 1246-1261 (2017).

13. Qiu, W. L. et al. Deciphering Pancreatic Islet beta Cell and alpha Cell Maturation Pathways and 463 Characteristic Features at the Single-Cell Level. Cell Metab 25, 1194-1205 e4 (2017).

14. Zeng, C. et al. Pseudotemporal Ordering of Single Cells Reveals Metabolic Control of Postnatal 465 beta Cell Proliferation. Cell Metab 25, 1160-1175 e11 (2017).

15. Krentz, N. A. J. et al. Single-Cell Transcriptome Profiling of Mouse and hESC-Derived 467 Pancreatic Progenitors. Stem Cell Rep. 11, 1551-1564 (2018).

16. Terskikh, A. et al. 'Fluorescent timer': protein that changes color with time. Science 290, 1585469 8 (2000).

17. Miyatsuka, T., Li, Z. \& German, M. S. Chronology of islet differentiation revealed by temporal cell labeling. Diabetes 58, 1863-8 (2009).

18. Cormack, B. P., Valdivia, R. H. \& Falkow, S. FACS-optimized mutants of the green fluorescent protein (GFP). Gene 173, 33-8 (1996). 
19. Xiao, X. et al. No evidence for beta cell neogenesis in murine adult pancreas. J Clin Invest 123, 2207-17 (2013).

20. Miyatsuka, T., Matsuoka, T. A. \& Kaneto, H. Transcription factors as therapeutic targets for diabetes. Expert Opin Ther Targets 12, 1431-42 (2008).

21. Miyatsuka, T. Uncovering the mechanisms of beta-cell neogenesis and maturation toward development of a regenerative therapy for diabetes. Diabetol. Int. 6, 261-267 (2015).

22. Kataoka, K. et al. MafA is a glucose-regulated and pancreatic beta-cell-specific transcriptional activator for the insulin gene. J Biol Chem 277, 49903-10 (2002).

23. Matsuoka, T. A. et al. Members of the large Maf transcription family regulate insulin gene transcription in islet beta cells. Mol Cell Biol 23, 6049-62 (2003).

24. Olbrot, M., Rud, J., Moss, L. G. \& Sharma, A. Identification of beta-cell-specific insulin gene transcription factor RIPE3b1 as mammalian MafA. Proc Natl Acad Sci U A 99, 6737-42 (2002).

25. Matsuoka, T. A. et al. The MafA transcription factor appears to be responsible for tissue-specific expression of insulin. Proc Natl Acad Sci U A 101, 2930-3 (2004).

26. Butler, A., Hoffman, P., Smibert, P., Papalexi, E. \& Satija, R. Integrating single-cell transcriptomic data across different conditions, technologies, and species. Nat. Biotechnol. 36, $411-420$ (2018).

27. Stuart, T. et al. Comprehensive Integration of Single-Cell Data. Cell 177, 1888-1902.e21 (2019). 
28. van der Maaten, L. \& Hinton, G. Visualizing data using t-SNE. J Mach Learn Res 9, 2579-2605 (2008).

29. Qiu, X. et al. Single-cell mRNA quantification and differential analysis with Census. Nat. Methods 14, 309-315 (2017).

30. Trapnell, C. et al. The dynamics and regulators of cell fate decisions are revealed by pseudotemporal ordering of single cells. Nat. Biotechnol. 32, 381-386 (2014).

31. Chen, Y.-C., Taylor, A. J. \& Verchere, C. B. Islet prohormone processing in health and disease. Diabetes Obes. Metab. 20 Suppl 2, 64-76 (2018).

32. Nair, G. G. et al. Recapitulating endocrine cell clustering in culture promotes maturation of

33. Rezania, A. et al. Reversal of diabetes with insulin-producing cells derived in vitro from human pluripotent stem cells. Nat Biotechnol 32, 1121-33 (2014).

34. Pagliuca, F. W. et al. Generation of functional human pancreatic beta cells in vitro. Cell 159, 42839 (2014).

35. Gu, G. et al. Global expression analysis of gene regulatory pathways during endocrine pancreatic development. Development 131, 165-79 (2004).

36. Scavuzzo, M. A. et al. Endocrine lineage biases arise in temporally distinct endocrine progenitors during pancreatic morphogenesis. Nat. Commun. 9, 3356 (2018). 
510 37. Miettinen, P. J. et al. Impaired migration and delayed differentiation of pancreatic islet cells in $511 \quad$ mice lacking EGF-receptors. Dev. Camb. Engl. 127, 2617-2627 (2000).

512 38. Greiner, T. U., Kesavan, G., Stahlberg, A. \& Semb, H. Rac1 regulates pancreatic islet 513 morphogenesis. BMC Dev Biol 9, 2 (2009).

514 39. Yoshitomi, H. \& Zaret, K. S. Endothelial cell interactions initiate dorsal pancreas development 515 by selectively inducing the transcription factor Ptfla. Development 131, 807-17 (2004).

516 40. Lammert, E., Cleaver, O. \& Melton, D. Induction of pancreatic differentiation by signals from blood vessels. Science 294, 564-7 (2001).

518 41. McCarthy, D. J., Campbell, K. R., Lun, A. T. L. \& Wills, Q. F. Scater: pre-processing, quality 519 control, normalization and visualization of single-cell RNA-seq data in R. Bioinforma. Oxf. Engl. 33, 1179-1186 (2017). 


\section{Acknowledgements}

and M.S. German (University of California San Francisco, CA, USA) for their helpful advice and

criticism, and S. Takebe, H. Hibino and S. Ishikawa for their assistance with the experiments. We also

work was supported by JSPS KAKENHI (No. 16K09766, 19H04060), the Takeda Science

Disorders, and Eli Lilly Research Foundation (all to T.Mi.). Fellowship support was provided by the

JDRF Postdoctoral Fellowship award (to S.S.).

\section{Author contributions}

M.H., and H.Wi. contributed to the acquisition and analysis of the data. T.Ma., I.S., H.Wa., and F.C.L.

\section{Competing interests}

The authors declare no competing interests. 
541 Figure 1. Improved "Timer" fluorescent system for labeling newly generated $\boldsymbol{\beta}$ cells

542 (A) Schematic diagram of the concept for improved temporal labeling in Ins1-GFP; Timer (DsRed543 E5) double-transgenic mice. Whereas green fluorescence in newly generated $\beta$ cells of Ins 1-Timer 544 embryos is below the microscopic detection threshold, green fluorescence from Ins 1-GFP mice is 545 high enough to be detected by microscopy in newly generated $\beta$ cells as well as in mature $\beta$ cells. 546 Therefore, Ins1-GFP; Timer double-transgenic mice enable the labeling of newly generated $\beta$ cells 547 as green-fluorescent cells by microscopy, which can be distinguished from mature $\beta$ cells with green 548 and red fluorescence. (B) Fluorescence images of the pancreas of Ins1-Timer and Ins1-GFP; Timer 549 embryos at E16.5. Whereas all green-fluorescent cells exhibited red fluorescence in Ins1-Timer 550 embryos, green fluorescent cells without red fluorescence (arrows) were observed in Ins1-GFP; 551 Timer double-transgenic embryos. Scale bar, $50 \mu \mathrm{m}$. (C) Time-lapse imaging of fluorescent cells 552 within an E14.5 pancreatic bud of Ins 1-GFP; Timer embryos. Enlarged images of a green-fluorescent 553 cell at the starting point (white arrow) are shown in the right panels. Red fluorescence started to 554 appear 6 hours after the detection of green fluorescence. Scale bar, $50 \mu \mathrm{m}$. (D) Percentage of green555 dominant cells among all fluorescent cells in Ins 1-GFP; Timer embryos and neonates. Data represent 556 the mean \pm SEM. $\mathrm{n}=3-4$ for each group.

Figure 2. Expression pattern of key transcription factors in fluorescent cells of Ins1-GFP; Timer mice

560 (A-C) Immunofluorescence staining for PDX1, NKX6.1 and MAFA was performed in the pancreas of E16.5 Ins1-GFP; Timer embryos. Arrows indicate PDX1 (A), NKX6.1 (B) and MAFA (C)-positive green fluorescent cells. Scale bar, $20 \mu \mathrm{m}$. (D) Percentage of MAFA-positive cells among greenfluorescent newborn $\beta$ cells (green bars) and green/red double-fluorescent differentiated $\beta$ cells 
564 (yellow bars). Data represent the mean \pm SEM. ${ }^{*} p<0.05$ versus newborn $\beta$ cells; $\mathrm{n}=3-6$ for each 565 group.

Figure 3. Spatial heterogeneity of newly generated $\beta$ cells

568 (A) The pancreas of an Ins 1-GFP; Timer embryo was dissected at E16.5 and stained with DBA lectin, 569 a marker of pancreatic ducts (white). Whereas some green-fluorescent cells are attached to the duct cells (arrows; $\beta^{\text {duct }}$ cells), others exist apart from the duct cells (arrowheads), adjacent to pre-existing $\beta$ cells. Scale bar, $20 \mu \mathrm{m}$. (B) Three-dimensional images of $\beta$ cells in Ins1-GFP; Timer embryos at E18.5. Green-dominant cells without red fluorescence are observed (arrows), located away from the ductal structures (white). (C) The pancreas of Ins 1-GFP; Timer embryo was dissected at E16.5 and stained with DBA lectin (blue) and anti-PECAM1 antibody, a marker of blood vessels (white). There are green-fluorescent cells adjacent to blood vessels (arrows; $\beta^{\text {vessel }}$ cells), but away from the duct cells. Scale bar, $20 \mu \mathrm{m}$. (D) Percentage of $\beta^{\text {duct }}$ cells and $\beta^{\text {vessel }}$ cells among total fluorescent cells in the pancreata of Ins1-GFP; Timer embryos and neonates. Data represent the mean \pm SEM. $* p<0.05$ versus $\beta^{\text {vessel }}$ cells; $\mathrm{n}=3-5$ for each group.

Figure 4. Single-cell transcriptome analysis for fluorescent cells of Ins1-GFP; Timer mice

(A) FACS plot of E16.5 Ins 1-GFP; Timer pancreatic cells used for library generation. (B) t-SNE plot of all three libraries; two green fluorescent (G1, G2) and one green/red fluorescent (GR) cell populations. (C-D) Pseudotime trajectory of G1, G2 and GR cells plotted bypseudotime (C) and 584 library identities (D). (E) t-SNE plot of 5 cell clusters from E16.5 endocrine-lineage cells in green585 fluorescent cells: $G^{\text {Neurog3 }}$ (purple), $G^{S s t}$ (brown), $G^{G c g}$ (blue), $G^{P d x l}$ (pink) and $G^{I n s 2}$ (green) cells. (F) Top ten differentially expressed genes in the 5 endocrine-lineage clusters. 
589 (A) Minimal spanning tree of pseudotime analysis for $G^{\text {Neurog3 }}$ (purple), $G^{\text {Sst }}$ (brown), $G^{G c g}$ (blue), $590 G^{P d x 1}$ (pink) and $G^{\text {Ins2 }}$ (green) cells generated by Monocle. Pseudotime from 0 to 15 orders $G^{\text {Neurog3 }}$ 591 cells first, following by $G^{S s t}$, and then $G^{G c g}$ and $G^{P d x l} / G^{I n s 2}$ cells. (B) Single cell gene expression of 592 Neurog3, Sst, Gcg, $P d x 1$ and Ins2 on the minimal spanning tree and mRNAs expression dynamics on $593 G^{G c g}$ and $G^{P d x l} / G^{I n s 2}$ branches over pseudotime. (C) Top one hundred differentially expressed genes 594 on $G^{G c g}$ and $G^{P d x l} / G^{I n s}$ branches generated by branched expression analysis modeling. (D) Gene 595 ontology analysis for $G^{\text {Ins2 }}$ cells by Panther.

Figure 6. Single-cell transcriptome analysis for late maturing $\beta$ cells

(A) t-SNE plot of 6 cell clusters from E16.5 green/red-fluorescent cells: $G R^{S p p l}$ (deep green), $G R^{P d x I}$

(green), GR $R^{S y t 4}$ (pink), GR $R^{N p y}$ (brown), $G R^{S c g 2}$ (blue) and $G R^{S p c 25}$ (purple) cells. (B) Top ten differentially expressed genes in the 6 green/red-fluorescent cell clusters. (C)pseudotime trajectory of $G R^{S p p 1}, G R^{P d x 1}, G R^{S y t l 4}, G R^{N p y}, G R^{S c g 2}$ and $G R^{S p c 25}$ cells. Pseudotime from 0 to 30 orders $G R^{S p p 1}$, $G R^{P d x 1}, G R^{S y t l 4}, G R^{N p y}, G R^{S c g 2}$ cells and makes a branching point to replicating $G R^{S p c 25}$ cells before going to $G R^{N p y} / G R^{S c g 2}$ cells. (D)pseudotime trajectory plotted by pseudotime. (E) Percentage of cells on S, G2/M and G1 cell cycle stages in each cluster cells. Note no G1 cells detected in $G R^{S p c 25}$ cluster.

Figure 7. Heterogeneous expression pattern of transcription factors by location of newly generated $\beta^{\text {duct }}$ cells and $\beta^{\text {vessel }}$ cells

(A-F) Immunofluorescence staining for Gcg, Mafb and Mafa was performed in the pancreas of E16.5 609 Ins1-GFP; Timer embryos. (A) Arrows indicate Gcg-positive green fluorescent cells. Arrowheads 610 indicate Gcg-positive green/red double fluorescent cells. (C) Arrows indicate Mafb-positive green 611 fluorescent cells. (E) Arrows indicate Mafa-positive green fluorescent cells. (B, D, F) Percentage of 612 Gcg (B), Mafb (D), Mafa (F)-positive cells among $\beta^{\text {duct }}$ cells or $\beta^{\text {vessel }}$ cells. Scale bar, $20 \mu$ m. Data 613 represent the mean \pm SEM. $* p<0.05 ; \mathrm{n}=3-6$ for each group. 
$615 \quad$ Figure 8. Single-cell transcriptome analysis for human $\beta$-like cells

616 (A) t-SNE plot of 7 cell clusters from day 27 human $\beta$-like cells: $h \beta^{S S T}$ (pink), $h \beta^{G C G}$ (brown), $h \beta^{G A S T}$ 617 (purple), $h \beta^{M G S T 1}$ (red), $h \beta^{S Y T 13}$ (blue), $h \beta^{I A P P}$ (deep green) and $h \beta^{N E F M}$ (green) cells. (B) Top ten 618 differentially expressed genes in the 7 human $\beta$-like cell clusters. (C) Single cell gene expression of $619 I N S, G C G, S S T, M A F B, M G S T 1$ and SYT13. (D)pseudotime trajectory of $h \beta^{S S T}, h \beta^{G C G}, h \beta^{G A S T}$, $620 h \beta^{M G S T 1}, h \beta^{S Y T 13}, h \beta^{I A P P}$ and $h \beta^{N E F M}$ cells. Pseudotime from 0 to 15 orders $h \beta^{S S T}$ first, following $h \beta^{G C G}$, $621 h \beta^{G A S T}, h \beta^{I A P P}$ and $h \beta^{N E F M}$ cells. $h \beta^{M G S T 1}$ and $h \beta^{S Y T 13}$ cells are branched from $h \beta^{G A S T}$ and $h \beta^{I A P P}$ cell, 622 respectively. (E) Pseudotime trajectory plotted by pseudotime.

\section{SUPPLEMENTAL FIGURE LEGENDS}

625 Figure S1. Improved detection of newly generated $\beta$ cells in Ins1-GFP; Timer reporter mice through their increased fluorescence intensity, related to Fig. 1

(A) Schematic diagram of the concept for improved temporal labeling in Ins 1-GFP; Timer doubletransgenic mice. As the intensity of green fluorescence is below the microscopic detection threshold in Ins 1-Timer (DsRed-E5) embryos, green-fluorescent $\beta$ cells can be detected only by flow cytometry.

630 In contrast, the green fluorescence from Ins 1-GFP mice is sufficiently high to be detected by 631 microscopy, and hence Ins1-GFP; Timer double transgenic mice enable us to label newly generated $632 \beta$ cells as green-fluorescent cells, which can be detected by microscopy separately from mature $\beta$ 633 cells with green and red fluorescence. (B) The pancreas was dissected from an Ins 1-GFP; Timer 634 embryo at E17.5 and stained with anti-insulin antibody. All fluorescent cells were positive for insulin. 635 Scale bar, $50 \mu \mathrm{m}$.

636

637 Figure S2. Location analysis of newly generated $\boldsymbol{\beta}$ cells by three-dimensional images, related to $638 \quad$ Fig. 3 
639 Three-dimensional images of an Ins1-GFP; Timer embryo at E18.5, stained with DBA lectin (blue), 640 and with anti-PECAM1 antibody (white). Green-fluorescent cells were observed adjacent to the blood 641 vessels and away from the duct cells (arrows).

Figure S3. Single-cell transcriptome analysis for green- and green/red-fluorescent cells, related 644 to Fig. 4

645 (A) t-SNE plot of 12 clusters from E16.5 green- and green/red-fluorescent cells: insulin-expressing $646 \beta$-cell lineage clusters $\left(\beta^{S p p 1}, \beta^{\text {Neurog3 }}, \beta^{S s t}, \beta^{G c g}, \beta^{P d x 1}, \beta^{I n s 1}, \beta^{N p y}\right.$ and $\left.\beta^{S p c 25}\right)$, duct, acinar, stellate and 647 endothelial cells. (B) Top ten differentially expressed genes in the $8 \beta$-cell lineage clusters. (C) Single 648 cell gene expression of Ins 1 and Ins2. (D) Pseudotime trajectory of $8 \beta$-cell lineage clusters $\left(\beta^{S p p 1}\right.$, $649 \beta^{\text {Neurog3 }}, \beta^{S s t}, \beta^{G c g}, \beta^{\text {Pdxl }}, \beta^{\text {Ins } 1}, \beta^{\text {Npy }}$ and $\left.\beta^{\text {Spc25 }}\right)$. Pseudotime from 0 to 40 orders $\beta^{\text {Spp } 1}, \beta^{\text {Neurog3 }}, \beta^{S s t}, \beta^{G c g}$, $650 \beta^{P d x I}, \beta^{I n s 1}, \beta^{N p y}$ cells and makes a branching point to replicating $\beta^{S p c 25}$ cells before going to $\beta^{N p y}$ cells. 651 (E) Single cell gene expression of Ins 1 and Ins 2 on the minimal spanning tree. (F) Ins 1 and Ins 2 gene 652 expression dynamics on the pseudotime trajectory over pseudotime.

Figure S4. Single-cell transcriptome analysis for green-fluorescent cells, related to Fig. 4 and 655 Fig. 5

656 (A) t-SNE plot of 8 cell clusters from E16.5 green-fluorescent cells: endocrine (Endo1 and Endo2), trunk, duct, stellate, endothelial, replicating (Rep), and low number gene (Low nGene) cells. Only endocrine (Endo1 and Endo2) cluster cells are used for further analysis in Fig. 4E, 4F and Fig. 5A-E. 660 
663 (A) Single cell gene expression of Neurog3, Sst, Gcg, $P d x 1$ and Ins2 on t-SNE plot of 5 cell clusters 664 from E16.5 endocrine-lineage cells in green-fluorescent cells (related to Fig, 4E and 4F). (B-D) Single 665 cell gene expression on the minimal spanning tree and gene expression dynamics on $G^{G c g}$ and $666 G^{P d x l} / G^{I n s 2}$ branches over pseudotime. Transcription factors important for pancreas development 667 (Mafb, Isll and Arx in B), ER stress-associated highly expressed genes in $G^{I n s 2}$ (Calr, Hspa5 and $668 S d f 2 l 1$ in C) and highly expressed genes in $G^{P d x l}$ (Mgstl and Syt13 in D) are shown, related to Fig. 669 5A-E. (E) Gene ontology analysis for $G^{P d x I}$ cells by Panther.

Figure S6. Integration analysis of mouse embryonic $\beta$-cell lineage cells and human $\beta$-like cells (A) t-SNE plot of 14 clusters from E16.5 green- and green/red-fluorescent cells in Ins 1-GFP; Timer mice, integrated with day 27 human $\beta$-like cells: insulin-expressing $\beta$-cell lineage clusters $\left(c \beta^{G C G}\right.$, $c \beta^{S S T}, c \beta^{N E U R O G 3}, c \beta^{I N S}, c \beta^{N E F M}, c \beta^{P C S K 1} c \beta^{G C G}$, and $\left.c \beta^{S P P I}\right)$, duct, acinar, stellate, endothelial, replicating (Rep), and miscellaneous (Misc) cells. (B) Top ten differentially expressed genes in the 8 $\beta$-cell lineage clusters. (C) Single cell gene expression of NEUROG3, SST, GCG and INS. (D) t-SNE plot grouped by species: human (pink) and mouse (blue) cells. $c \beta^{G C G}, c \beta^{S S T}$ and $c \beta^{N E U R O G 3}$ clusters are reflected by dotted lines. (E) Proportions of human and mouse cells in each cluster cells. $c \beta^{G C G}$, $c \beta^{S S T}$ and $c \beta^{N E U R O G 3}$ clusters (surrounded by dotted line) contain more than $15 \%$ of human or mouse cells.

\section{Figure S7. Spatial heterogeneity of $\beta$-cell neogenesis revealed by Ins1-GFP; Timer embryos}

683 Proposed model for $\beta$-cell neogenesis. Endocrine progenitors differentiate into two types of $\beta$ cells; $684 \beta^{\text {duct }}$ and $\beta^{\text {vessel }}$ cells. The $\beta^{\text {duct }}$ cells are observed in the ductal region where Neurog3-expressing endocrine progenitors emerge. In contrast, part of endocrine progenitors migrate away from the ductal area toward the region near the blood vessels and differentiate into $\beta^{\text {vessel }}$ cells. 
689 Table S1. Top ten differentially expressed genes in the 4 non- $\beta$-cell lineage clusters of E16.5 690 green- and green/red-fluorescent cells, related to Figure S3

691

692 Movie S1. Ex vivo real-time imaging of the developing pancreas of Ins1-GFP; Timer mice

693 The developing pancreata of Ins 1-GFP; Timer embryos at E14.5 were dissected and cultured ex vivo 694 to acquire real-time imaging of fluorescent cells. Images were acquired every $30 \mathrm{~min}$ for a total of 10 695 hours. 


\section{Figures}

A

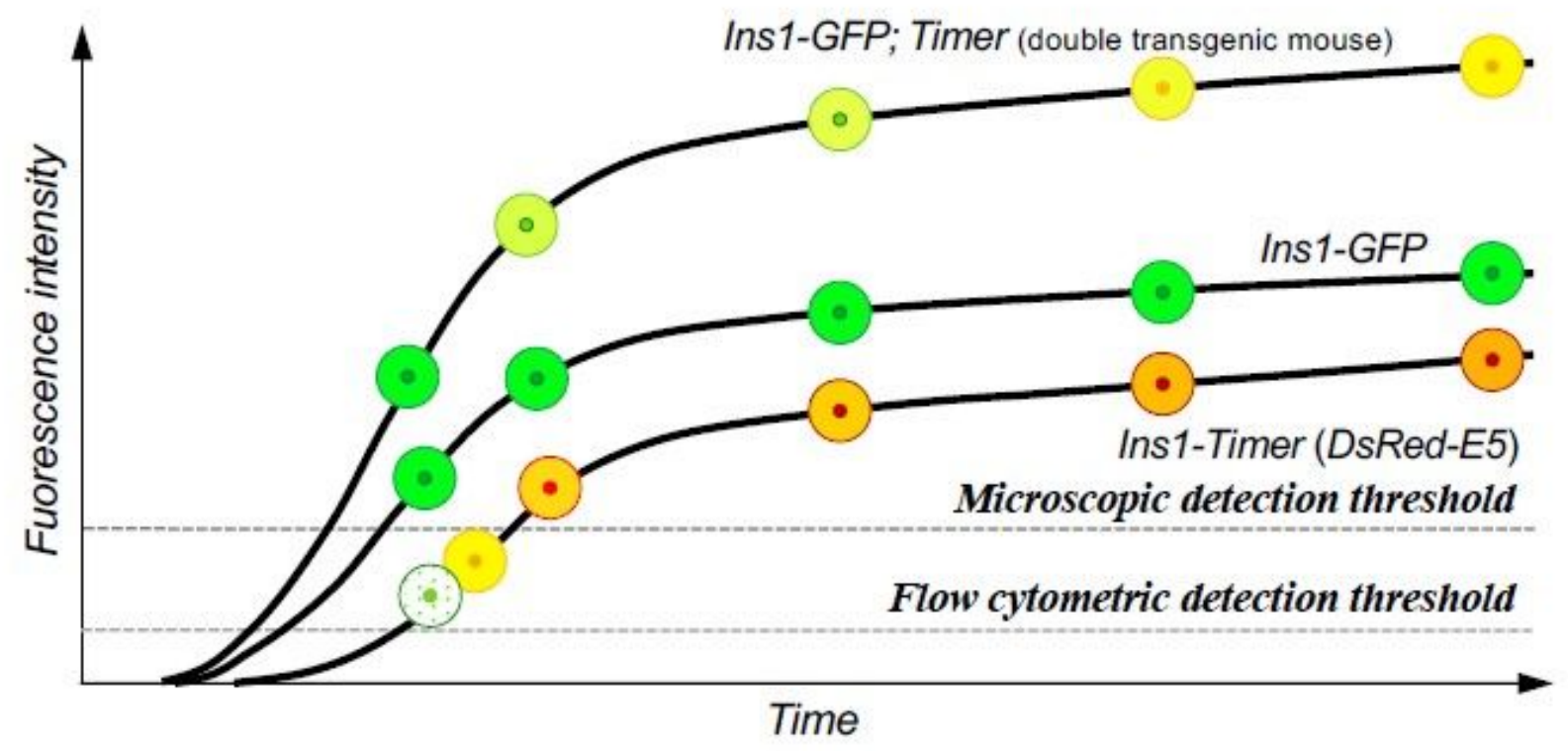

$\mathrm{B}$

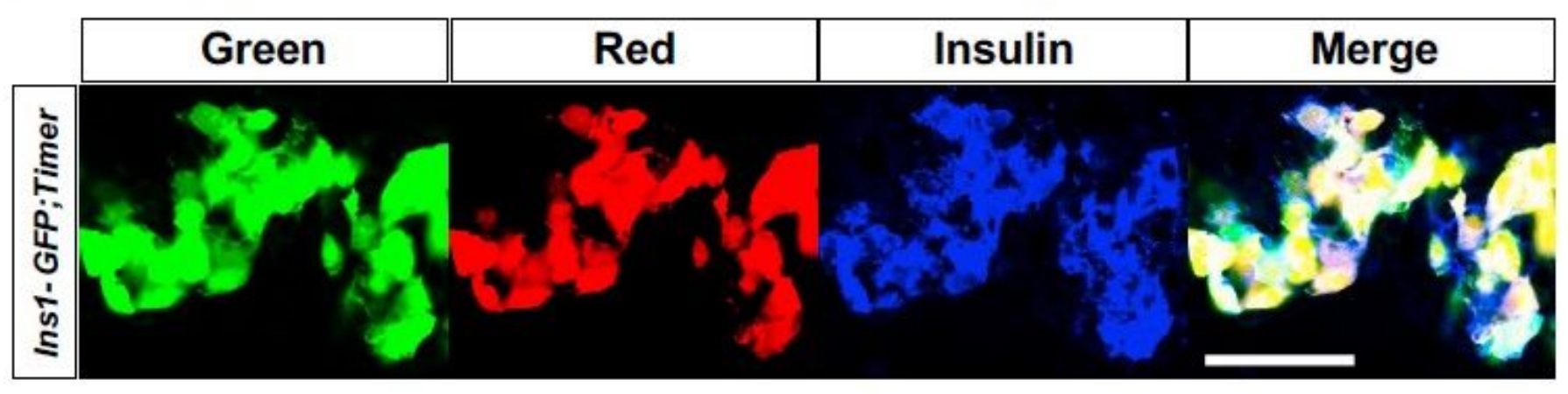

Figure 1

Improved "Timer" fluorescent system for labeling newly generated $\beta$ cells (A) Schematic diagram of the concept for improved temporal labeling in Ins1-GFP; Timer (DsRed- E5) double-transgenic mice. Whereas green fluorescence in newly generated $\beta$ cells of Ins1-Timer embryos is below the microscopic detection threshold, green fluorescence from Ins1-GFP mice is high enough to be detected by microscopy in newly generated $\beta$ cells as well as in mature $\beta$ cells. Therefore, Ins1-GFP; Timer double-transgenic mice enable the labeling of newly generated $\beta$ cells as green-fluorescent cells by microscopy, which can be distinguished from mature $\beta$ cells with green and red fluorescence. (B) Fluorescence images of the pancreas of Ins1-Timer and Ins1-GFP; Timer embryos at E16.5. Whereas all green-fluorescent cells exhibited red fluorescence in Ins1-Timer embryos, green fluorescent cells without red fluorescence (arrows) were observed in Ins1-GFP; Timer double-transgenic embryos. Scale bar, $50 \mu \mathrm{m}$. (C) Time-lapse imaging of fluorescent cells within an E14.5 pancreatic bud of Ins1-GFP; Timer embryos. Enlarged 
images of a green-fluorescent cell at the starting point (white arrow) are shown in the right panels. Red fluorescence started to appear 6 hours after the detection of green fluorescence. Scale bar, $50 \mu \mathrm{m}$. (D) Percentage of green dominant cells among all fluorescent cells in Ins1-GFP; Timer embryos and neonates. Data represent the mean \pm SEM. $n=3-4$ for each group.

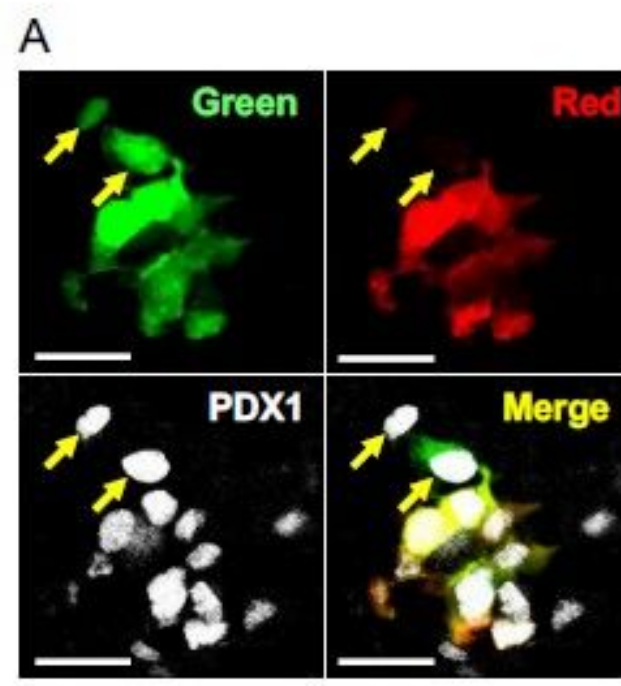

B

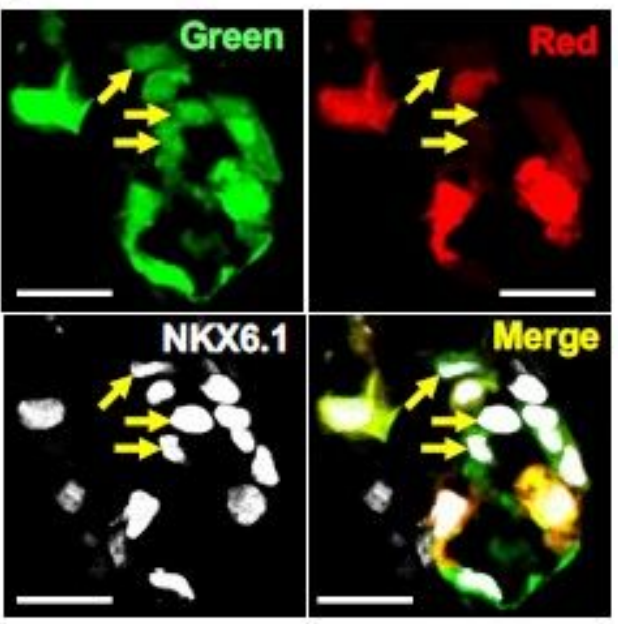

C
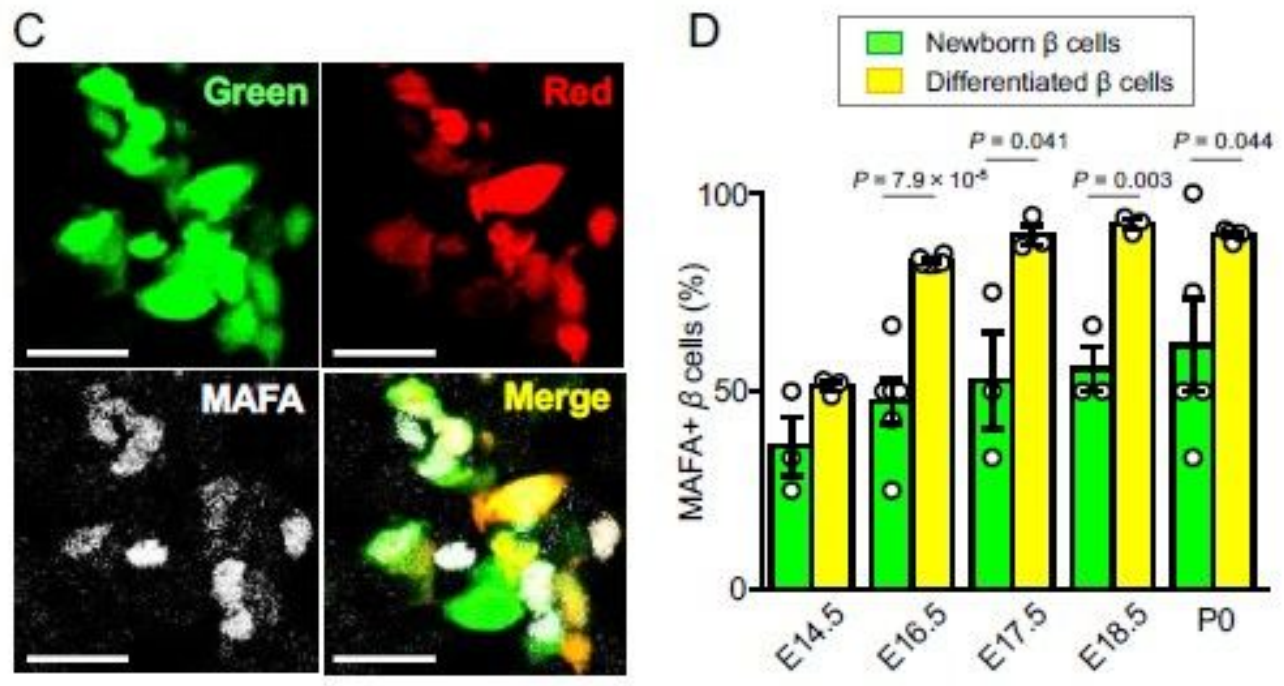

Figure 2

Expression pattern of key transcription factors in fluorescent cells of Ins1-GFP; Timer mice (A-C) Immunofluorescence staining for PDX1, NKX6.1 and MAFA was performed in the pancreas of E16.5 Ins1GFP; Timer embryos. Arrows indicate PDX1 (A), NKX6.1 (B) and MAFA (C)-positive green fluorescent cells. Scale bar, $20 \mu \mathrm{m}$. (D) Percentage of MAFA-positive cells among green fluorescent newborn $\beta$ cells (green bars) and green/red double-fluorescent differentiated $\beta$ cells (yellow bars). Data represent the mean \pm SEM. ${ }^{*} p<0.05$ versus newborn $\beta$ cells; $n=3-6$ for each group. 
A

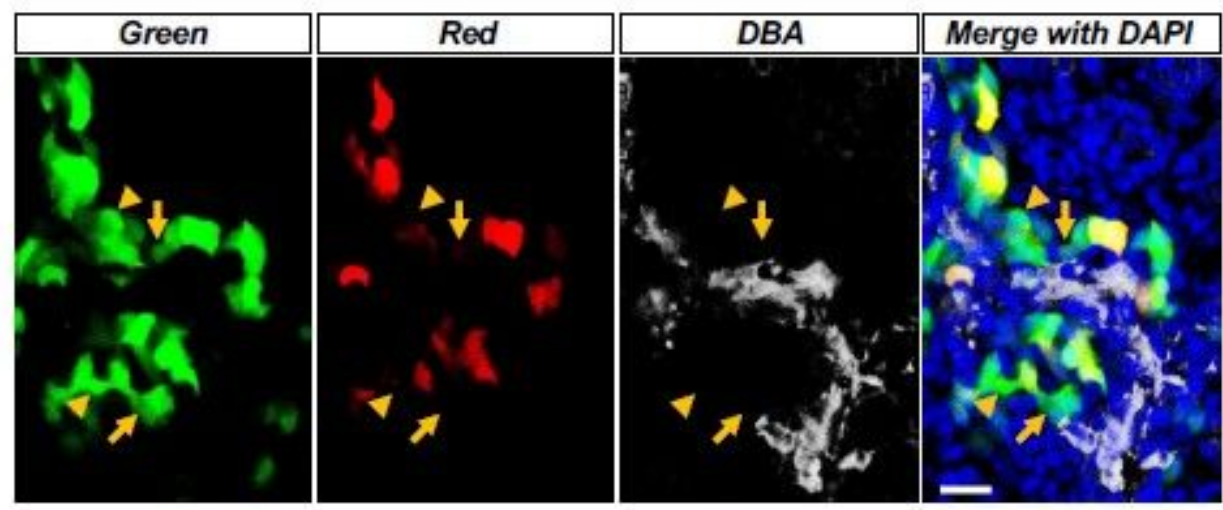

B

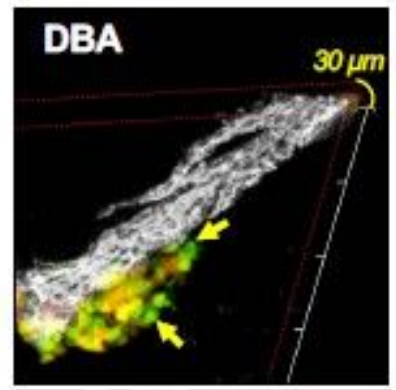

C
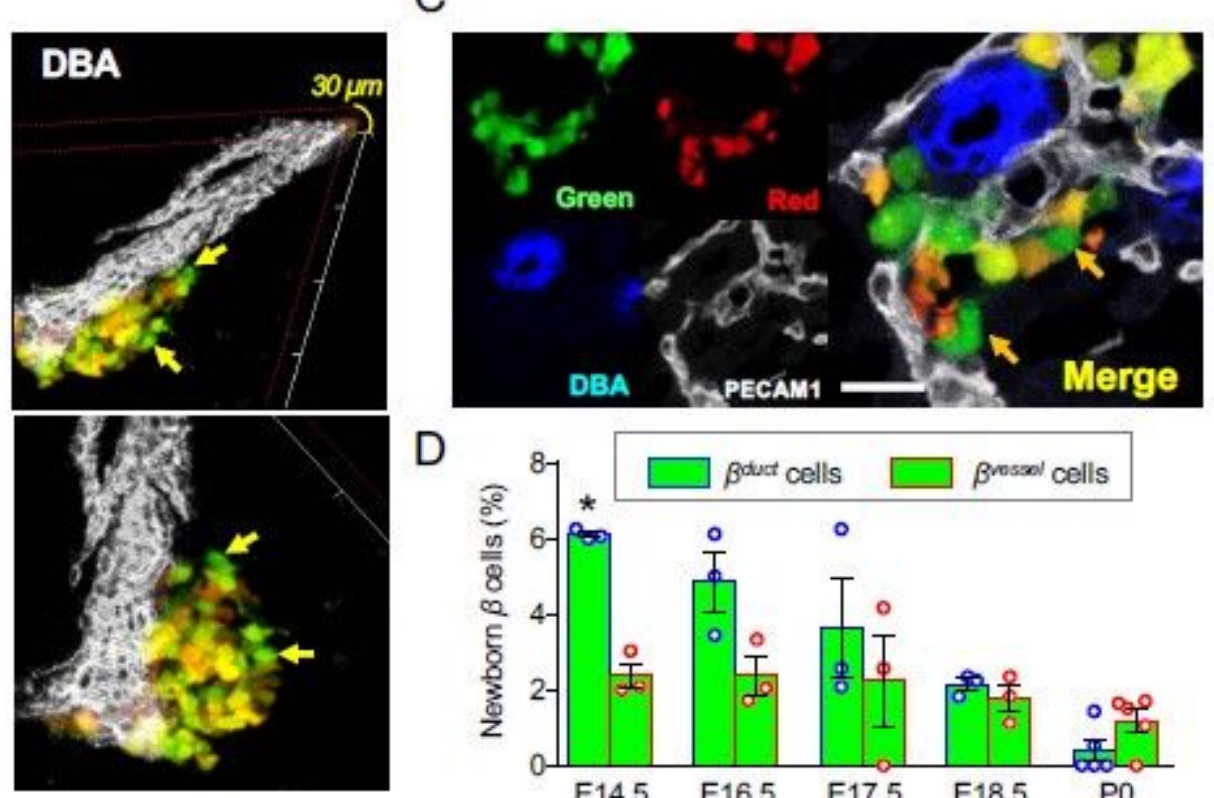

D

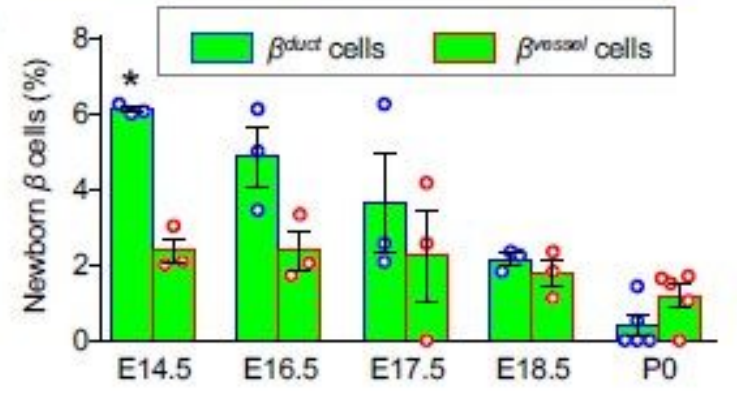

Figure 3

Spatial heterogeneity of newly generated $\beta$ cells (A) The pancreas of an Ins1-GFP; Timer embryo was dissected at E16.5 and stained with DBA lectin, a marker of pancreatic ducts (white). Whereas some green-fluorescent cells are attached to the duct cells (arrows; $\beta$ duct cells), others exist apart from the duct cells (arrowheads), adjacent to pre-existing $\beta$ cells. Scale bar, $20 \mu \mathrm{m}$. (B) Three-dimensional images of $\beta$ cells in Ins1-GFP; Timer embryos at E18.5. Green-dominant cells without red fluorescence are observed (arrows), located away from the ductal structures (white). (C) The pancreas of Ins1-GFP; Timer embryo was dissected at E16.5 and stained with DBA lectin (blue) and anti-PECAM1 antibody, a marker of blood vessels (white). There are green-fluorescent cells adjacent to blood vessels (arrows; $\beta$ vessel cells), but away from the duct cells. Scale bar, $20 \mu \mathrm{m}$. (D) Percentage of $\beta$ duct cells and $\beta$ vessel cells among total fluorescent cells in the pancreata of Ins1-GFP; Timer embryos and neonates. Data represent the mean \pm SEM. ${ }^{*} p<0.05$ versus $\beta$ vessel cells; $n=3-5$ for each group. 


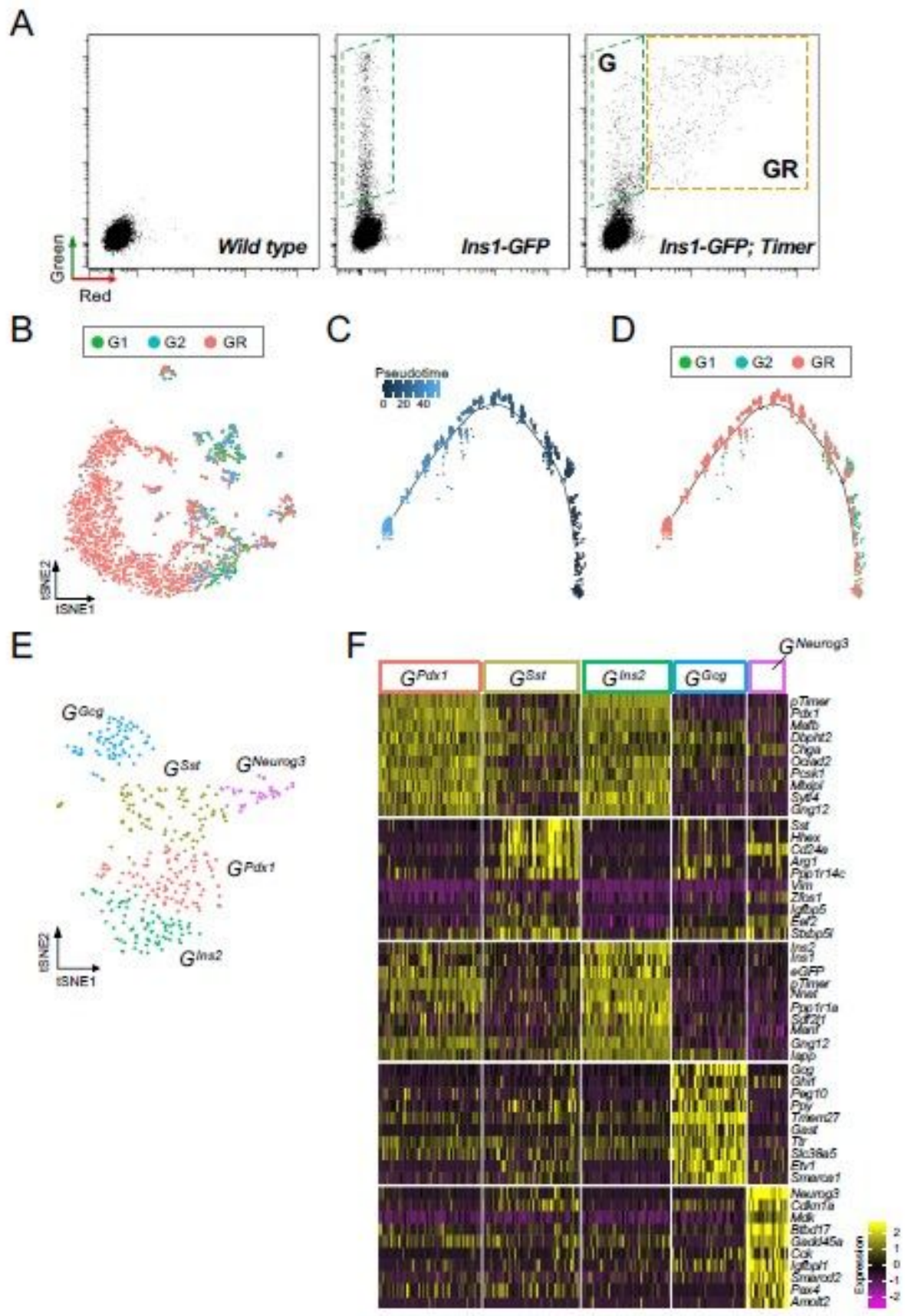

\section{Figure 4}

Single-cell transcriptome analysis for fluorescent cells of Ins1-GFP; Timer mice (A) FACS plot of E16.5 Ins1-GFP; Timer pancreatic cells used for library generation. (B) t-SNE plot of all three libraries; two green fluorescent $(G 1, G 2)$ and one green/red fluorescent (GR) cell populations. (C-D) Pseudotime trajectory of G1, G2 and GR cells plotted bypseudotime (C) and library identities (D). (E) t-SNE plot of 5 cell clusters from E16.5 endocrine-lineage cells in green fluorescent cells: GNeurog3 (purple), GSst (brown), GGcg (blue), GPdx1 (pink) and GIns2 (green) cells. (F) Top ten differentially expressed genes in the 5 endocrinelineage cluster 


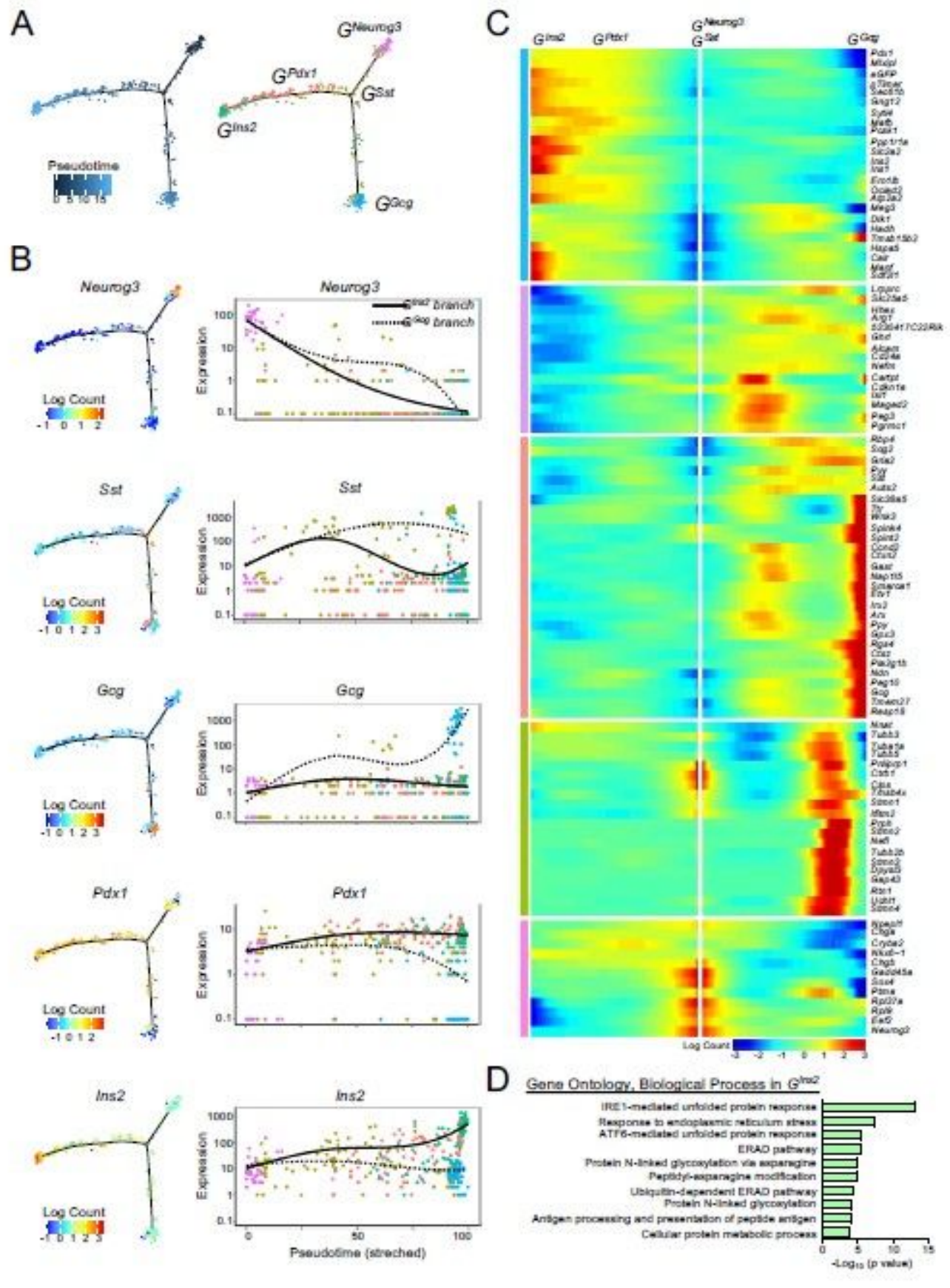

\section{Figure 5}

Single-cell transcriptomic dynamics at branching point in early $\beta$-cell specification (A) Minimal spanning tree of pseudotime analysis for GNeurog3 589 (purple), GSst (brown), GGcg (blue), GPdx1 (pink) and GIns2 (green) cells generated by Monocle. Pseudotime from 0 to 15 orders GNeurog3 cells first, following by GSst, and then GGcg and GPdx1/GIns2 cells. (B) Single cell gene expression of Neurog3, Sst, Gcg, $\mathrm{Pdx} 1$ and Ins2 on the minimal spanning tree and mRNAs expression dynamics on GGcg and GPdx1/GIns2 branches over pseudotime. (C) Top one hundred differentially expressed genes on GGcg and GPdx1/GIns2 branches generated by branched expression analysis modeling. (D) Gene ontology analysis for GIns2 cells by Panther. 
A
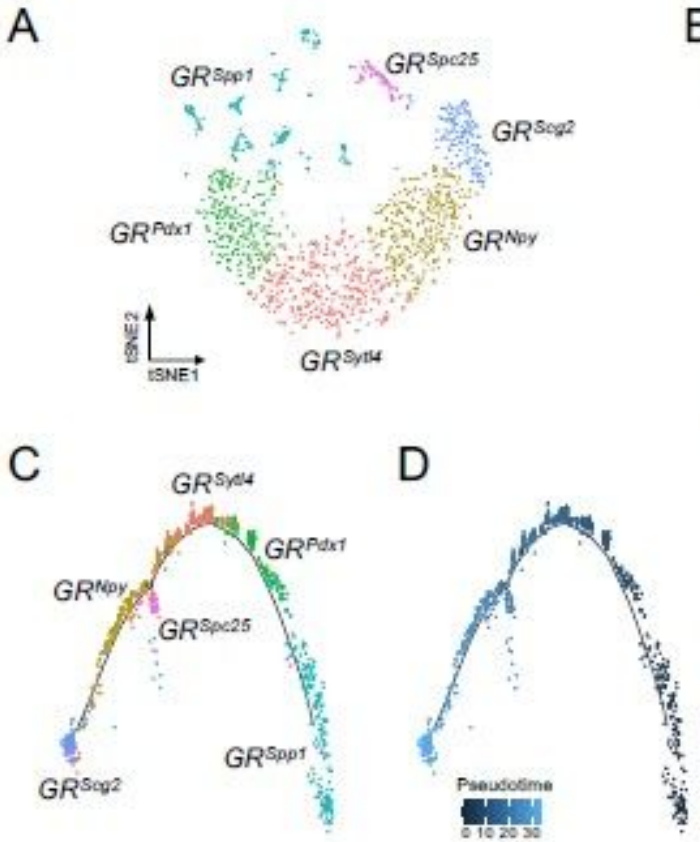

$\mathrm{E}$

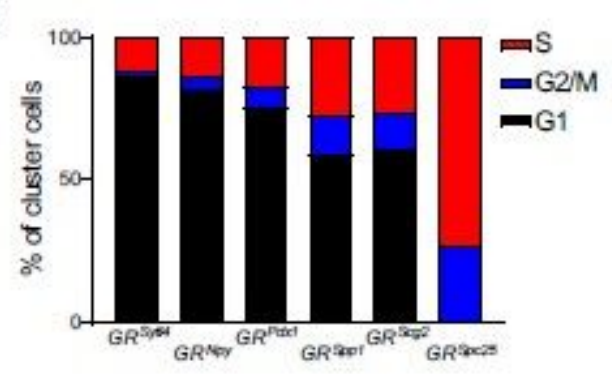

B

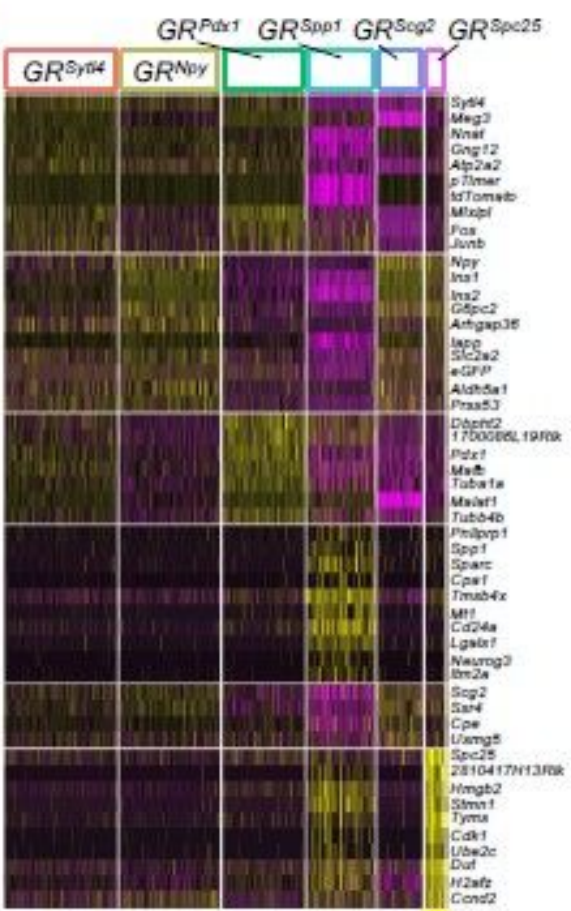

\section{Figure 6}

Single-cell transcriptome analysis for late maturing $\beta$ cells (A) t-SNE plot of 6 cell clusters from E16.5 green/red-fluorescent cells: GRSpp1 (deep green), GRPdx1 (green), GRSytl4 (pink), GRNpy (brown), GRScg2 (blue) and GRSpc25 (purple) cells. (B) Top ten differentially expressed genes in the 6 green/redfluorescent cell clusters. (C)pseudotime trajectory of GRSpp1, GRPdx1, GRSytl4, GRNpy, GRScg2 and GRSpc25 cells. Pseudotime from 0 to 30 orders GRSpp1, GRPdx1, GRSyt|4, GRNpy, GRScg2 cells and makes a branching point to replicating GRSpc25 cells before going to GRNpy/GRScg2 cells.

(D)pseudotime trajectory plotted by pseudotime. (E) Percentage of cells on S, G2/M and G1 cell cycle stages in each cluster cells. Note no G1 cells detected in GRSpc25 cluster. 

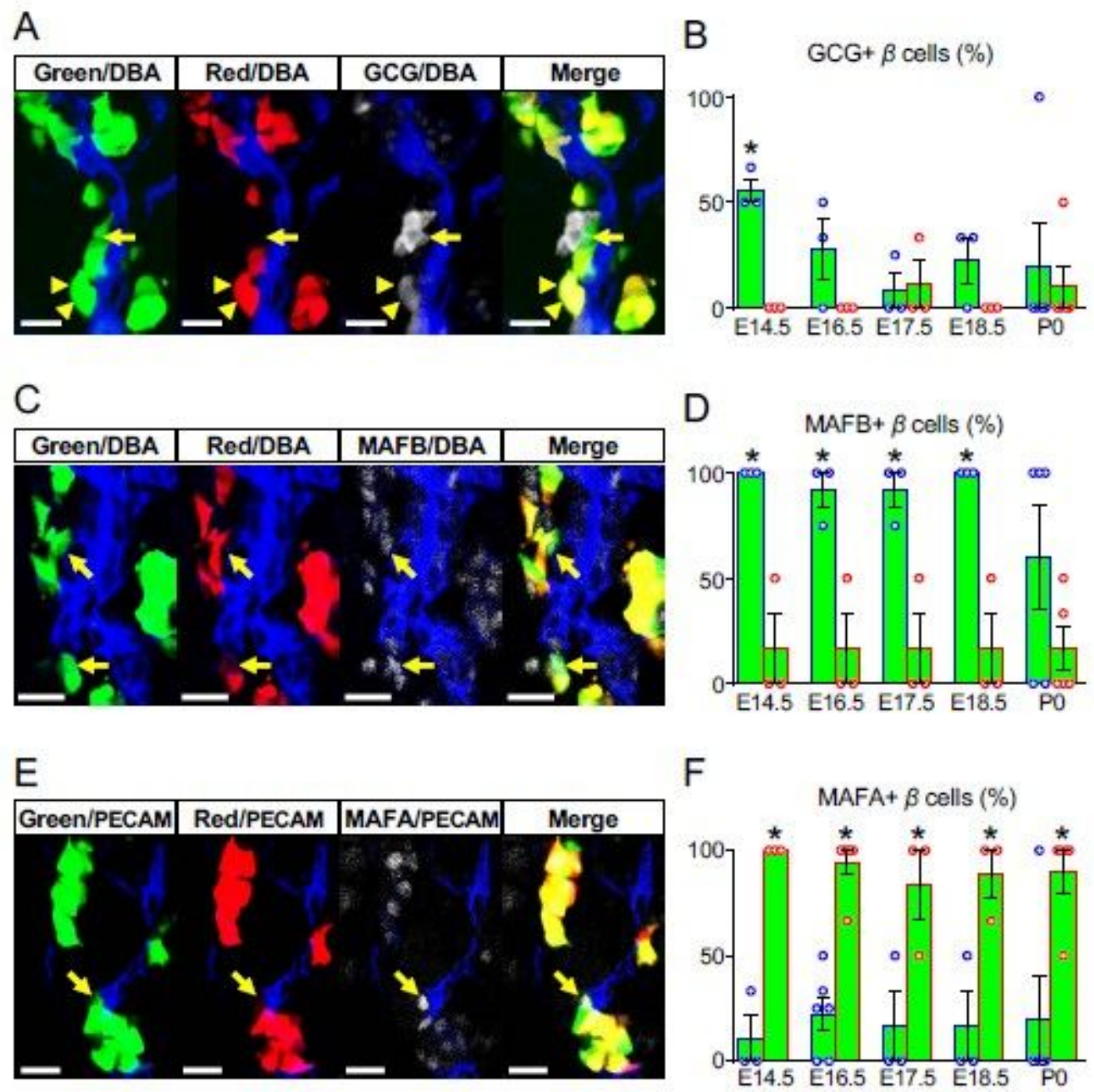

$$
\beta \text { duct cells }
$$

Bvossal cells

\section{Figure 7}

Heterogeneous expression pattern of transcription factors by location of newly generated $\beta$ duct cells and Bvessel cells (A-F) Immunofluorescence staining for Gcg, Mafb and Mafa was performed in the pancreas of E16.5 Ins1-GFP; Timer embryos. (A) Arrows indicate Gcg-positive green fluorescent cells. Arrowheads indicate Gcg-positive green/red double fluorescent cells. (C) Arrows indicate Mafb-positive green fluorescent cells. (E) Arrows indicate Mafa-positive green fluorescent cells. (B, D, F) Percentage of Gcg (B), Mafb (D), Mafa (F)-positive cells among $\beta$ duct cells or $\beta$ vessel cells. Scale bar, $20 \mu \mathrm{m}$. Data represent the mean \pm SEM. * $p<0.05 ; n=3-6$ for each group. 


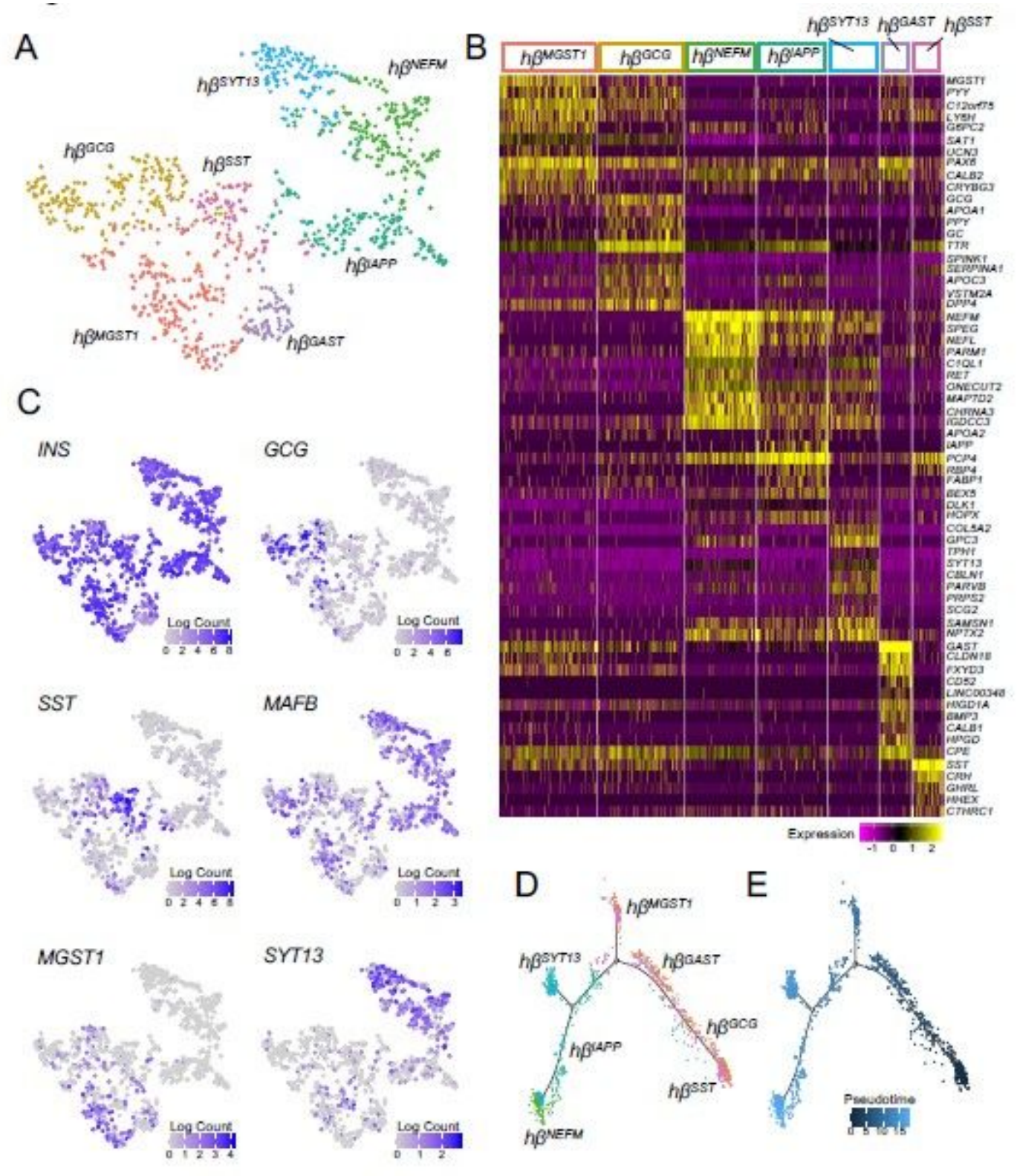

\section{Figure 8}

Single-cell transcriptome analysis for human $\beta$-like cells (A) t-SNE plot of 7 cell clusters from day 27

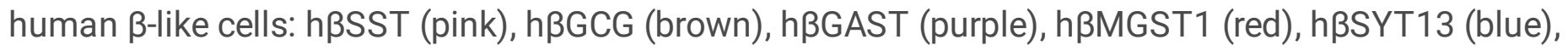
h $\beta$ IAPP (deep green) and hßNEFM (green) cells. (B) Top ten differentially expressed genes in the 7 human $\beta$-like cell clusters. (C) Single cell gene expression of INS, GCG, SST, MAFB, MGST1 and SYT13.

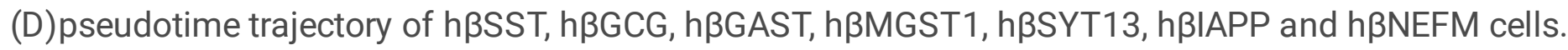

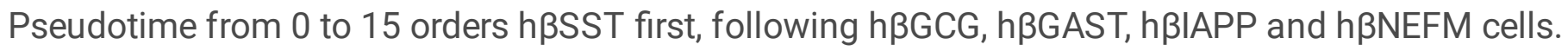
$h \beta M G S T 1$ and $h \beta S Y T 13$ cells are branched from $h \beta G A S T$ and hßIAPP cell, respectively. (E) Pseudotime trajectory plotted by pseudotime.

\section{Supplementary Files}


This is a list of supplementary files associated with this preprint. Click to download.

- FigureS1.jpg

- Figures2.jpg

- FigureS3.jpg

- FigureS4.jpg

- FigureS5.jpg

- FigureS6.jpg

- FigureS7.jpg

- TableS1.png 